\title{
Le théâtre dans le monde arabe
}

\author{
Eve FEUILLEBOIS
}

Sorbonne Nouvelle - Paris 3, UMR 7528 Mondes iranien et indien

Il serait présompteux de prétendre présenter ici la création théâtrale de la vingtaine d'états qui forment le monde arabe. Nous nous bornerons donc à examiner les productions théâtrales des pays du Moyen-Orient et de l'Afrique du Nord ; les expériences théâtrales plus marginales des pays de la péninsule arabique, de la Jordanie, de la Lybie, du Soudan et des Comores ne seront pas abordées.

\section{Le théâtre et la tradition littéraire arabe}

\section{A. Y a-t-il absence de tradition théâtrale dans le monde arabe classique?}

On entend généralement dire que l'héritage littéraire arabe pré-moderne n'offre pas d'exemples de théâtre ressemblant à ce que l'Occident entend par ce mot. Dans Studies in the Arab Theatre and Cinema, Jacob M. Landau affirme que le théâtre moderne est un «produit totalement étranger transplanté dans la terre vierge des sociétés arabes » et qu'il n'y a pas eu de théâtre arabe avant le XIX ${ }^{\mathrm{e}}$ siècle et l'invasion de l'Égypte par Napoléon (Landau, 1958 : XII).

La position du volume collectif publié par Nadia Tomiche (Le Théâtre arabe, 1969) est plus nuancée : si Jacques Berque estime que le monde arabe classique est dépourvu de théâtre tout en mettant en garde contre un certain orientalisme prompt à en déduire une incompatibilité entre l'Islam et le théâtre (Tomiche, 1969 :15-16), Cherif Khaznadar estime que le théâtre est un phénomène populaire et universel et présente, tout au long de son article, les formes et aspects de l'expression dramatique arabe traditionnelle (Tomiche, $1969: 39 s q$ ). Quant à Jean Duvignaud, il affirme que si toutes les sociétés connaissent des formes de dramatisation spontanées, cela ne signifie pas qu'elles deviennent systématiquement des formes artistiques réelles ou contribuent à l'apparition de ce mode de création hautement particulier que nous avons coutume d'appeler théâtre. Il en profite pour réfuter deux malentendus extrêmement fréquents : la prétendue incompatibilité entre le théâtre et l'islam, 
et la thèse d'un commencement tardif et sous influence occidentale du «théâtre » dans cette région du monde (Tomiche, 1969 : 194-197).

Par contre, dans l'introduction à son anthologie, Short Arabic Plays, Salma Khadra Jayyûsî écrit que le théâtre arabe «a peu été pratiqué et est demeuré dans l'obscurité » pendant des siècles, ne devenant un genre majeur qu'au XX $\mathrm{X}^{\mathrm{e}}$ siècle (Jayyûsî, 2003 : VIII). George Allen dans An Introduction to Arabic Literature (2000) indique que, si l'héritage littéraire arabe pré-moderne ne nous offre pas d'exemples d'œuvres théâtrales qui puissent être reliées à la tradition occidentale, il ne manque pas de genres indigènes possédant des qualités dramatiques.

Dans le numéro thématique d'Horizons maghrébins (Le théâtre arabe au miroir de luimême), l'existence de ces genres dramatiques traditionnels est reconnue, mais la qualité d'œuvre théâtrale leur est déniée. Abdelghani Maghnia affirme : « Il est absurde de croire qu'il y ait pu y avoir du théâtre dans l'ancienne société marocaine » (Maghnia, 2008 : 64). À la page suivante, Jean-François Clément trouve dangereuse la thèse soutenue par certains théoriciens maghrébins du théâtre selon laquelle le thêâtre aurait toujours existé dans les sociétés maghrébines et les accuse de confondre théâtralisation et théâtre, les formes traditionnelles de spectacle relevant d'un «préthéâtre » ou du «degré zéro du théâtre » (Clément, $2008: 65)$.

Quant à l'article de Monica Ruocco dans Histoire de la littérature arabe moderne, il contourne habilement le problème :

[...] la question de l'existence des pratiques théâtrales antérieures au développement de la dramaturgie moderne a été longtemps débattue par les historiens. La théorie la plus accréditée conclut que le monde arabophone a connu dans le passé certaines formes de spectacle, mais que ces manifestations n'ont pas été décisives dans l'implantation du théâtre contemporain (HallâqToelle, $2007: 151)$.

Pour elle, le théâtre arabe naquit au XIX ${ }^{\mathrm{e}}$ siècle dans le cadre de la Nahda dont l'un des efforts porta sur l'adaptation d'un répertoire étranger aux exigences d'un nouveau public, même s'il est indéniable que des formes pré- ou para-théâtrales influencèrent le développement de l'art dramatique arabe, lui donnant une identité originale.

La vraie question est de savoir ce que l'on entend par théâtre : s'agit uniquement du théâtre aristotélicien basé sur un texte et centré sur des acteurs humains représentant une histoire à travers une action et des dialogues, ou bien la définition peut-elle être élargie pour 
englober d'autres types de performances? Dans un article de The Drama Review, John Bell soupçonne ce déni de tradition théâtrale autochtone d'être une tentative de dévalorisation de la culture arabe (Bell, 2005 :10), certes largement inconsciente, mais qui a cependant réussi à investir l'esprit des Arabes eux-mêmes. Car des genres littéraires contenant des éléments dramatiques et des formes spectaculaires populaires existent bel et bien dans le monde arabe avant le XIXe siècle, mais aucun de ces phénomènes n'a été considéré comme relevant du théâtre.

Par contre, toutes sortes de raisons ont été invoquées pour tenter d'expliquer l'inexistence du théâtre dans la civilisation arabe. Des personnalités arabes et occidentales ont prétendu que la «structure mentale» des Arabes était incompatible avec cette forme d'art : l'esprit arabe, atomiste et individualiste, serait incapable de l'organisation élaborée et de la structuration nécessaires aux formes littéraires d'envergure, comme l'épopée ou le drame (Ben Halima). Indépendamment du fait que cette thèse découle d'un préjugé raciste, elle est contredite par les nombreuses réalisations intellectuelles (sciences religieuses, philosophie) et artistiques (architecture) des Arabes, impliquant toutes un esprit de synthèse et des facultés d'organisation exceptionnelles (Khozai, 1984 : 3 ; Badawi, 1988 : 5). Certains justifient cette absence par une mythologie insufisamment développée (And), comme si le théâtre ne pouvait éclore que du mythe.

Pour d'autres (Tawfîq al-Hakîm, Zaki Tulaymat, Al-Raï), des facteurs environnementaux, comme les rudes conditions météorologiques dans le désert et le nomadisme avec ses pérégrinations continuelles, ses razzias et ses guerres intestines, expliquent que le climat n'ait pas été propice à la naissance du théâtre, celui-ci ne se développant bien qu'en milieu urbain. Or, le monde arabe s'est sédentarisé et urbanisé dès la période omeyyade, encore plus sous les Abbassides, et même à la période préislamique il existait des centres urbains importants comme la Mekke et Ta'if (Khozai, 1984 : 6 ; Badawi, 1988 : 4). On a également invoqué l'interdiction faite aux femmes de se produire sur la scène (Landau), en oubliant que les théâtres grec, romain et médiéval recouraient au travestissement des acteurs pour les rôles féminins.

Les raisons d'ordre religieux ont été jugées convaincantes par certains chercheurs : le théâtre s'appuie sur l'existence d'un conflit (conflit entre la volonté humaine et la toutepuissance divine, entre l'individu et les valeurs ou lois d'une société, conflit interne, etc.) et le musulman ne connaîtrait pas ces types de conflit à cause de sa conception autocratique de Dieu et de sa soumission au destin (Aziza, Khozai). Nonobstant la compréhension assez simpliste de l'islam que cela implique, c'est réduire le théâtre à la seule tragédie, la comédie 
n'étant pas basée sur un conflit (Badawi, 1988: 4). D'autres invoquent l'interdit de la représentation : l'islam n'aurait pas toléré qu'on rivalisât avec Dieu, seul façonnier des images (Amin, Hunayn). Or le Coran et le hadith ne condamnent pas univoquement la peinture et la sculpture, et l'islam a d'ailleurs très tôt toléré les images (miniatures, fresques) lorsque tout soupçon d'idolâtrie était écarté (Khozai, 1984 : 8-13 ; Badawi, 1988 : 4).

Quelques-uns optent pour des raisons à la fois historiques et esthétiques (Sayed Attia Abul Naga). Dans le monde méditerranéen, la littérature dramatique était tombée en décadence pendant l'époque romaine et avait disparu. Les Arabes ne trouvèrent donc pas d'exemples vivants de cet art dans les pays dont ils firent la conquête. Certes, ils auraient pu s'inspirer du patrimoine théâtral légué par la Grèce antique comme ils furent profondément influencés par la philosophie et les sciences grecques, mais la critique littéraire privilégiait la poésie, seul art par excellence. Au X $\mathrm{X}^{\mathrm{e}}$ siècle, le texte de l'Ars poetica d'Aristote fut traduit du syriaque en arabe par Abû Bishr Mattâ et Yahyâ ibn 'Adî. Les commentaires de ce texte par Fârâbî, Avicenne et Averroès montrent clairement qu'ils n'avaient aucune familiarité avec ces formes inconnues dans leur culture : les deux premiers utilisent les termes grecs tout en faussant leur sens, et le troisième traduit «tragédie » par madîh (panégyrique) et « comédie » par hijâ (satire), des genres poétiques hautement prisés (Khozai, 1984 : 4-6).

\section{B. Des formes autochtones populaires}

Le monde arabe a connu, avant la période napoléonienne, un certain nombre de genres autochtones, certes très différents du théâtre occidental, mais possédant indubitablement des qualités dramatiques ; celles-ci n'ont malheureusement pas reçu l'attention qu'elles méritaient en raison des préjugés, mais aussi de l'indigence et du caractère indirect des sources : les témoignages sont rares concernant ces formes essentiellement orales. Il faut cependant signaler les travaux de Chérif Khaznadar, Mohamed Aziza, Mohammed Badawi, Ahmed Cheniki et surtout Shmuel Moreh (Live Theatre and Dramatic Literature in the Medieval Arab World, 1992).

Selon Chérif Khaznadar, des manifestations dramatiques existaient dès la période préislamique, dans les fêtes consacrées aux divinités ou les fêtes saisonnières de Nayrûz (printemps) et de Mihrajân (automne), à l'occasion des foires périodiques, dans les joutes oratoires entre poètes et les performances des «récitateurs » (muqallid). Si l'islam a éliminé la plupart des cérémonies rituelles comme les processions et les mascarades, il en a toléré certaines (le stambeli tunisien et le zâr égyptien [figure 118], qui sont pourtant des 
survivances de rites animistes d'Afrique noire), et en a suscité d'autres, notamment dans ses marges chiite ( $t a$ 'ziye) et soufie (rites confrériques des Mevlevis ou des Aïssaouas [figure 119]) (Tomiche, $1969: 43-44)$.

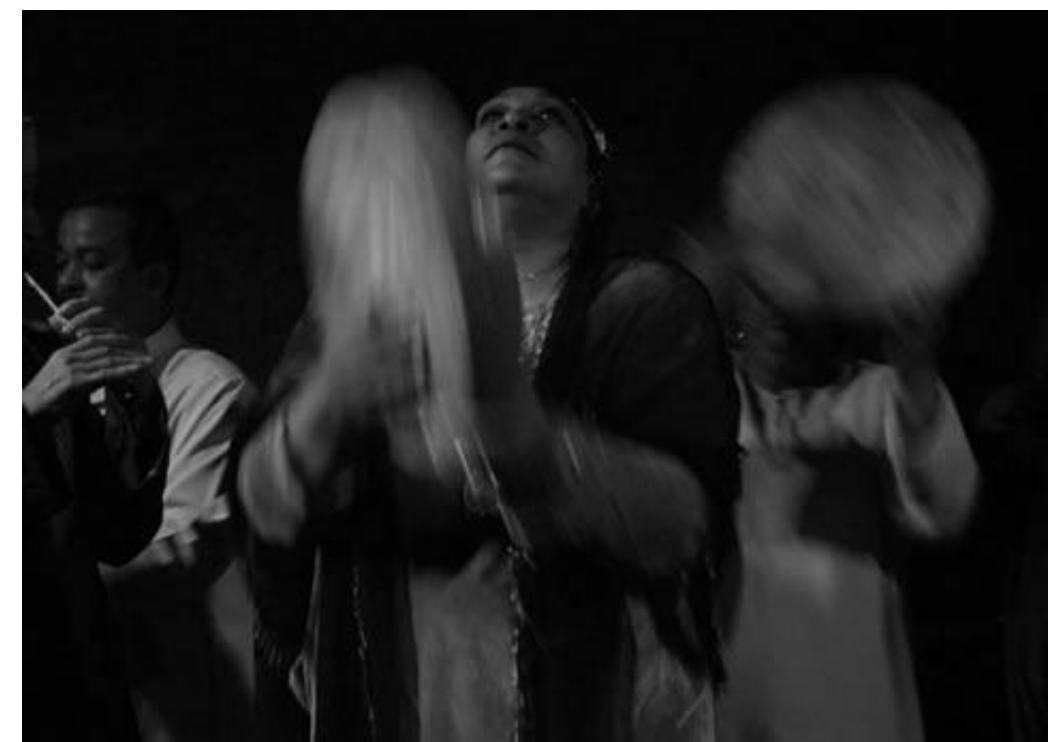

Figure 118 : Séance de zar égyptien, Genève 2009, photo Moustafa Ali, site Ateliers d'ethnomusicologie, URL : http://www.adem.ch/archives/transe09/mazaher.html

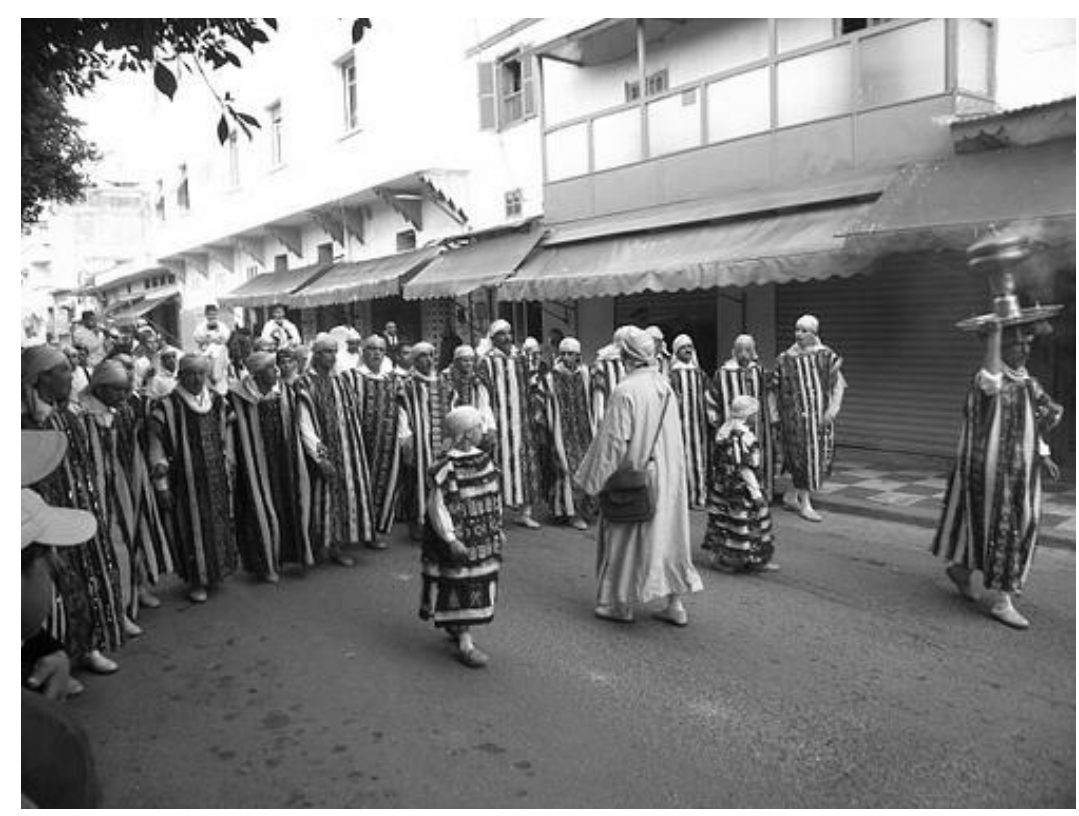

Figure 119 : Aissaoua : cérémonie de la confrérie à Sidi M'hamed Ben Aissa à Meknès http://www.meknassy.com/index.php?option=com_content\&task=view\&id=33\&Itemid=38 
Le monde arabe médiéval connaît des professionnels du divertissement tels que les la 'âbûn ${ }^{1}$ (clowns, bouffons, mimes), ou les mukhannathûn ${ }^{2}$ (musiciens, danseurs ou acteurs). Les divertissements de cour apparaissent lors du règne du calife Mutawakkil (r. 847-861). La musique et la poésie y tiennent évidemment une place de choix : la poésie est chantée, accompagnée d'instruments, et même parfois mimée. Par ailleurs, des spectacles comiques et des danses grotesques d'acteurs masqués, connus sous le nom de samâja, sont attestés en Égypte et en Iraq du $\mathrm{IX}^{\mathrm{e}}$ au XI $\mathrm{XI}^{\mathrm{e}}$ siècle : ces artistes se produisent à la cour et sur les marchés, se déguisent fréquemment en animaux, sont parfois confondus avec les mukhâyilûn, acteurs non masqués, et prennent le nom de muharrijûn au XIX ${ }^{\mathrm{e}}$ siècle.

Les fous ou bouffons (mudhikûn) sont connus dès l'époque prophétique : ils officient à la cour des grands, mais aussi chez les riches marchands à l'occasion de festivités, et sur les places de marché. À partir du XII ${ }^{\mathrm{e}}$ siècle, le mot maskhara se met à désigner l'art du bouffon qui invente des histoires pour rire (maqâmât) et récite des poèmes parodiques. Une autre tradition est celle des chars animés à l'occasion des mariages, circoncisions et autres fêtes : des artisans y mimaient leur activité, des acrobates, montreurs d'animaux, magiciens, jongleurs et autres musiciens s'y produisaient, les différents chars se suivant en procession (Moreh, 1992).

L'art du conteur peut aussi être considéré comme une performance. Les lexicographes arabes définissent hikâya comme signifiant l'imitation, la personnification, aussi bien que le récit. Le hâqi est donc le mime capable d'imiter les accents comme les manières de faire, et la hikâya une pièce dialoguée présentée par un ou des acteur(s) éventuellement déguisé(s). Ces artistes récitaient des épisodes de romans médiévaux comme al-Zâhir Baïbars ou 'Antara, dans un mélange de prose et de vers. On ignore de quand exactement date le début de ce phénomène qui est florissant à partir du XVII ${ }^{\mathrm{e}}$ siècle dans les cafés de quartiers. L'arabisant Edward W. Lane, qui séjourna au Caire au cours des années 1830, en distingue plusieurs catégories : les «bardes » (shu'arâ', litt. «poètes »), spécialistes de la Geste des Beni Hilâl, récitaient de mémoire en s'accompagnant d'un rebab à une corde; les «raconteurs » (muhaddithîn) se consacraient à la récitation du Roman de Baybars; les «Antaristes » ( 'anâtra), spécialistes du Roman d'Antar, lisaient à haute voix à partir d'un texte écrit (Lane, 1836 : II, 117-162). Les derniers conteurs urbains ont disparu entre les années 1950 et 1970, mais il subsiste encore aujourd'hui dans plusieurs pays arabes, en milieu rural ou bédouin,

\footnotetext{
${ }^{1}$ Ibn al-Nadim emploie le terme de talâ'ub pour désigner les drames religieux indiens, tandis que les traducteurs du syriaque et du copte traduisent theatron par mal'ab.

${ }^{2}$ Le premier sens du mot est «efféminé », «homosexuel» ou «prostitué mâle », mais le terme est parfois employé pour signifier acteur ou musicien, ou encore comme synonyme de $l a$ ' $a \hat{b} b$.
} 
une tradition de récitation de la Geste hilalienne, sous une forme entièrement versifiée, et sans aucun support écrit, le récitant étant généralement illettré. [figure 120]

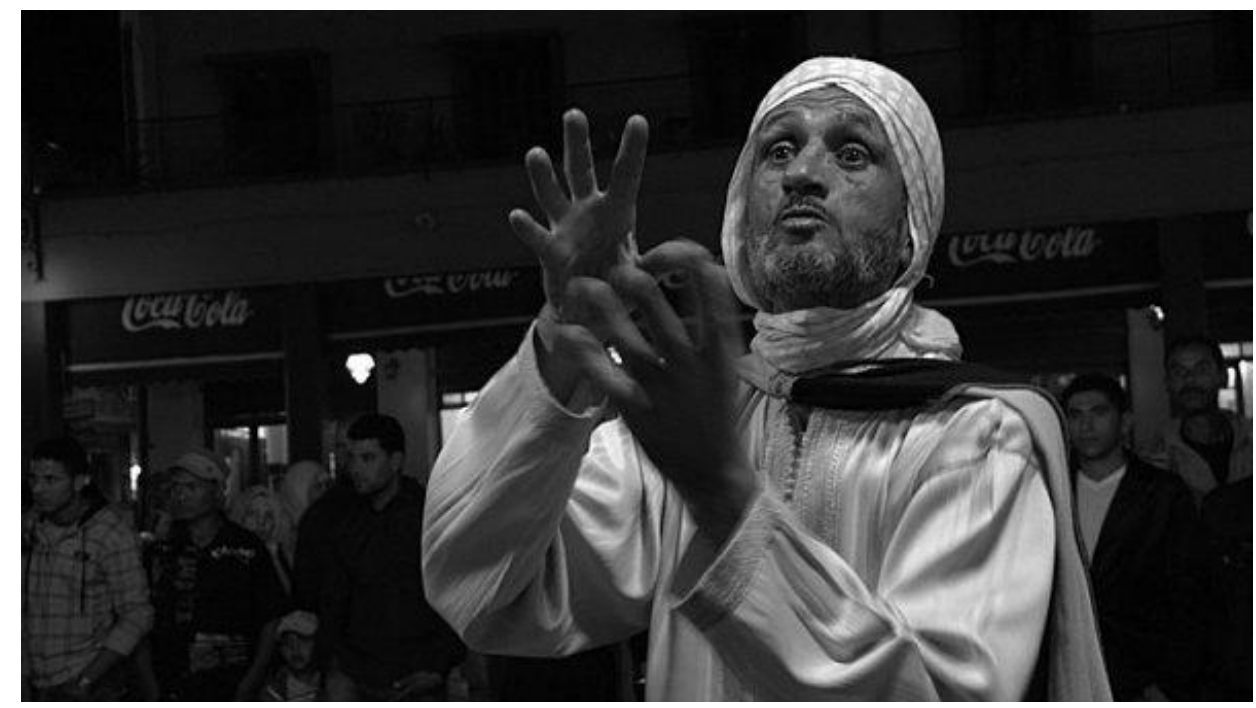

Figure 120 : Le célèbre conteur Abderrahim al-Makori, surnommé Al-Azaliyah sur la place J'maa elf'na, http://alhalqa.com/AlHalqaKinetics/francais/geschichtenerzaehler.html

De même, le khayâl est une sorte de théâtre vivant : le mot signifie d'abord la figure, ou la forme imaginaire, et ce n'est qu'au XI' siècle qu'il sera lié au mot zill («ombre »), pour signifier le théâtre d'ombres. Auparavant, il est joué par des acteurs ou imitateurs en chair et en os, et s'apparente à la hikâya. À partir du XVI ${ }^{\mathrm{e}}$ ou du XVII ${ }^{\mathrm{e}}$ siècle, un nouveau terme apparaît, celui de muhabbazun, qui désigne des amuseurs de quartier qui jouent des sketches comiques ou de courtes farces en rapport avec les réalités sociales et l'actualité, ce spectacle présentant des similarités avec l'orta oyunu turc et la Commedia dell'arte (Moreh, 1992). La plupart des témoignages nous sont parvenus d'Égypte, sous la forme de descriptions de voyageurs européens : ces farces assez simples et largement improvisées, jouées par des troupes ambulantes avec un minimum d'accessoires et de moyens, étaient volontiers satiriques ou truculentes (Badawi, $1988: 10-12$ ).

Pour Badawi, il faut voir dans la maqâma une écriture dramatique arabe originale : cette forme est au croisement du récit et du théâtre. Le théâtre d'ombres arabe a hérité de certaines des caractéristiques de ce genre lettré; d'ailleurs, les pièces de Muhammad ibn Daniyâl al-Mawsîlî (1248-1311) ont été qualifiées de maqâma par l'historien Ibn Iyâs (m. c. 1524) (Badawi, 1988: 5-6). Malheureusement peu de textes nous sont parvenus: nous disposons de trois pièces écrites par Ibn Daniyâl pour son ami le montreur d'ombres 'Alî ibn 
Mawlâhum, et intitulées L'Esprit de l'Ombre, L'Étrange prédicateur et l'étranger, Le Fou d'amour. Elles nous révèlent un art comique, satirique et souvent obscène, dont les personnages sont issus du bas de l'échelle sociale et présentés de manière humoristique : marginaux, escrocs à la petite semaine, entremetteuses, homosexuels. Le chant, la musique et la danse font partie intégrante du spectacle ; le texte mêle vers et prose rimée, langue savante et dialectal, dialogues et éléments descriptifs. Quelques textes du XVIII ${ }^{\mathrm{e}}$ siècle, attribués à Sa'ud, Shaykh 'Ali Nahla et Da 'ûd al-Manawî al-'Attâr, ont été sauvés de l'oubli par Hasan al-Qashshâsh (m. 1905) : écrits en dialectal égyptien, ils portent la marque de l'oralité et du folklore, sont composés en poésie strophique et destinés à être chantés. Si Ibn Daniyâl avait des prétentions artistiques, le théâtre d'ombres au XIX ${ }^{\mathrm{e}}$ siècle est devenu un art populaire, prisé des classes inférieures et joué dans les cafés (Badawi, 1988 : 11sqq; Khozai, 1984 :1924).

Certaines formes traditionnelles existent encore aujourd'hui, en particulier au Maghreb, ou n'ont disparu que récemment. En Tunisie, Oumouk Tangu est un rite incantatoire en temps de sécheresse, au cours duquel une poupée de chiffons est promenée par des petites filles. La Kharja de Sidi Bou Saïd est une procession en l'honneur d'un marabout ; l'initiation dans la confrérie des Aïssaouas s'accompagne de mime d'animaux sauvages et de tours de faqirs. Le stamboli de Sidi Saad est un rite sacrificiel suivi d'une cérémonie d'exorcisme et de possession, entrecoupée de sketches théâtraux. Le kalam malhoun oppose un récitant racontant en phrases rimées une aventure drôle et un chœur de jeunes gens relançant le récit par leurs interventions malicieuses. Nbitet el ballout («veillée du mensonge ») est une forme de sketche comique : Le Sultan et Zulaykha met en scène un sultan parodique, assis sur le trône et tenant une tomate, tandis que ses sujets se prosternent devant lui ; sa femme Zuleykha (dont le rôle est tenu par un homme travesti) l'assure qu'elle l'aime tout en le trahissant avec le poète Abu Nuwâs. Dans La Mère Balâza, le personnage principal est une entremetteuse, sage-femme peu farouche. La Tunisie connaît également les spectacles de marionnettes à fils originaires de Sicile (Aziza, $1975: 28 s q q$ ).

$\mathrm{Au}$ Maroc, Bu-Ilmaun («l'homme vêtu de peau ») était la dramatisation d'un ancien culte agraire, abondamment décrit au début du $\mathrm{XX}^{\mathrm{e}}$ siècle par les voyageurs européens et les officiers des affaires indigènes de l'armée coloniale, et étudié par Edmond Doutté et Émile Laoust. L'acteur, nu sous une peau de mouton, masqué d'une courge, doté d'un énorme phallus en érection et accompagné de personnages masqués grotesques, déambulait dans le village en mimant des scènes de la vie quotidienne et en se moquant des pouvoirs locaux. Le bsât' («divertissement») mettait en scène un homme déguisé en panthère et une femme- 
serpent-ogre, ainsi que des personnages représentant humoristiquement différents types sociaux ; il se composait de chants, de danses et de courtes saynètes satiriques. La fête de Soltân Tolba («roi des étudiants ») avait lieu chaque année au printemps à Fez et mettait en scène un sultan parodique pour une semaine, en souvenir du soutien apporté par les étudiants au sultan Moulay Rachid (1666-1672) [figure 121]. La Khalqa est un espace circulaire dans un lieu fréquenté où se produisent des acrobates, conteurs et musiciens qui divertissent un public de passage et vivent des quêtes. Sidi L'Katfi est une pratique dérivée des cérémonies des confréries soufies : elle se déroule dans un patio ou une grande pièce d'une maison de notables ; personnages et situations sont puisés dans les travers et vices des contemporains ; la représentation est précédée de la Hadra, une danse extatique (Badry-Cheniki in Corvin, $2008: 888 s q)$.

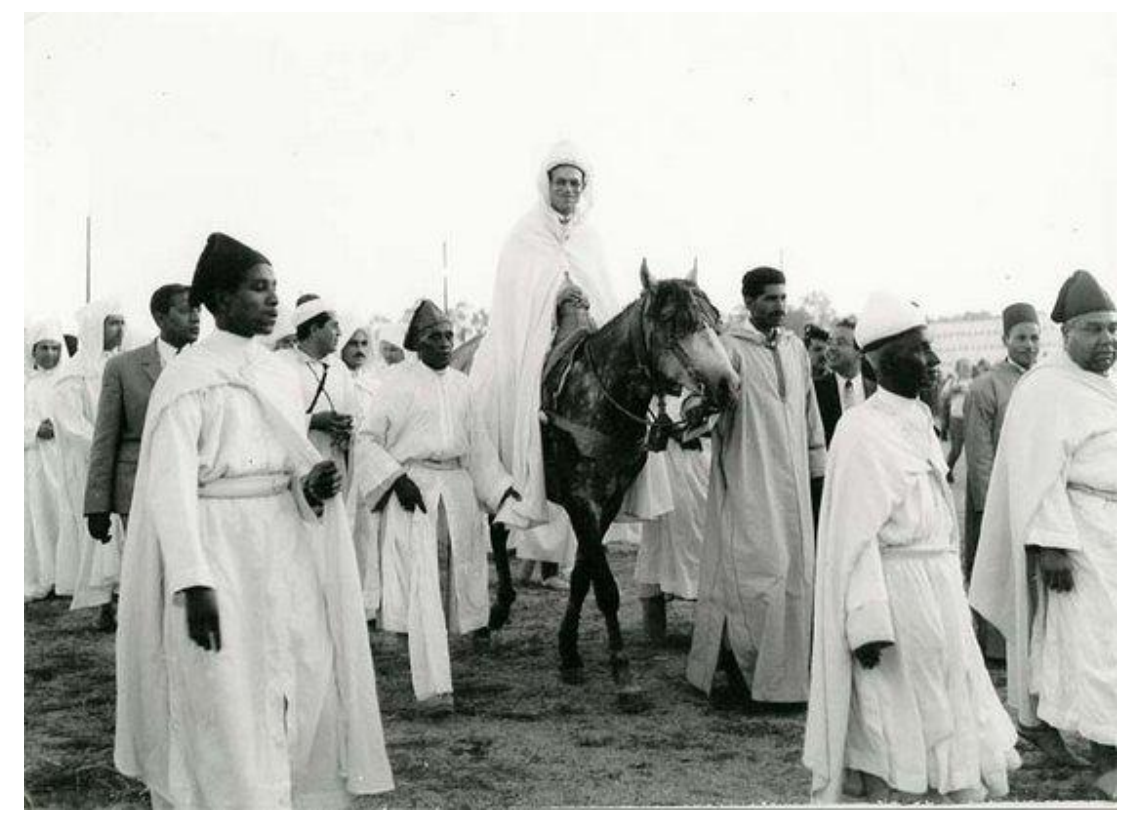

Figure 121 : Cérémonie de Soltan Tolba à Fès en 1967, photo Office marocain du tourisme ; site : Jewish Moroccan Heritage ; URL : http://www.judaisme-marocain.org/phototheque/visite.php?vue=icono\&id=2349\#; (C) Musée d'Art Juif Marocain, 2005.

Certaines fêtes comme celle de Sidi Mansour en grande Kabylie (Algérie) ressemblent aux dionysies grecques : des garçons déguisés ou travestis, mouchoirs à bout de bras, dansent et miment des personnages humains et des situations diverses (scènes de chasse au chacal, etc.). Ce spectacle s'articule autour de l'ouchen, héros des contes représenté par un jeune homme masqué d'une peau. En Algérie, les pèlerinages sont l'occasion de sketches burlesques représentant des scènes de ménage, et de tableaux satiriques mettant en scène des personnages truculents. Dans certaines villes d'Algérie et de Tunisie, deux conteurs aveugles accompagnés d'un bendir et d'un r'bab racontent dans un lieu public la vie du Prophète et de 
ses Compagnons. Entourés par les spectateurs, ils miment les personnages qu'ils évoquent et utilisent un bâton pour délimiter l'espace. Le bou-ghanim ou l'homme au roseau, joue et discute avec les arreddad, sorte de compagnons (Cheniki, 1993 : chapitre I) [figure 122].

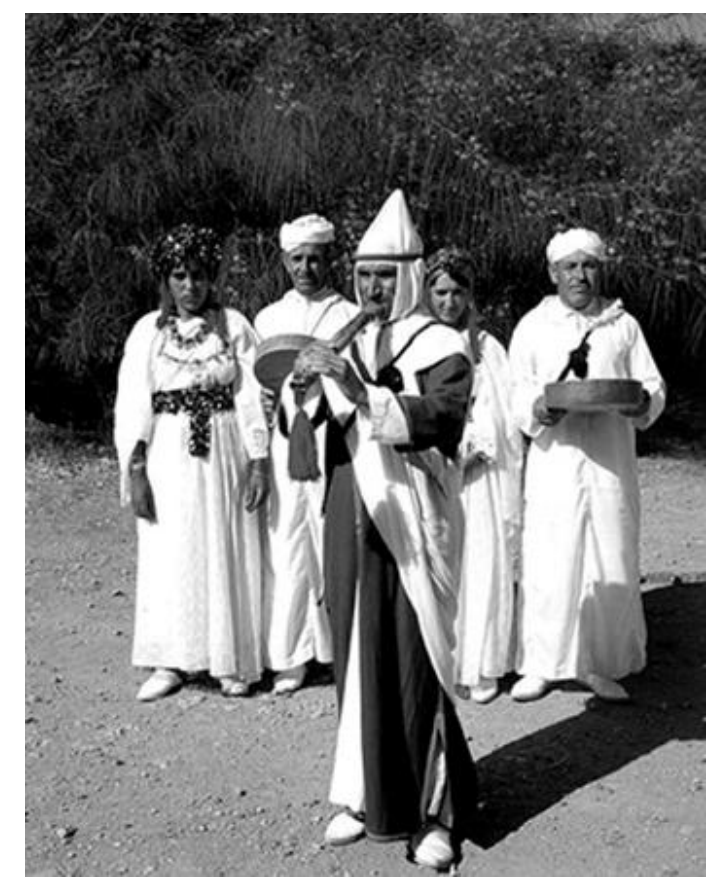

Figure 122 : L'ensemble de Boughanim des Aït al-Bouguemmaz, source : http://www.izlanzik.tv/modules/newbb/viewtopic.php?topic_id=156\&forum=13

\section{Définition du théâtre et idéologie}

Pourquoi, face à toutes ces formes traditionnelles, continuer à nier l'existence d'une «théâtralité » arabe ? Après tout, il ne vient à l'esprit de personne de dénier le caractère théâtral de formes comme le karagöz turc, le nô japonais ou les danses rituelles d'Asie du Sud-Est, ni de les qualifier de formes «primitives ». Pourquoi imposer aux seuls Arabes une définition aussi restrictive et aussi spécifique du théâtre, alors même que l'Occident n'a cessé, depuis le début $\mathrm{du} \mathrm{Xx}^{\mathrm{e}}$ siècle, de repousser les limites de cette définition pour lui-même ? La théâtralité, qui est une des dimensions universelles de la vie sociale, prend diverses formes dans divers contextes : pourquoi en attribuer le monopole à une forme occidentale, de surcroît datée ?

Cette conception, héritée des orientalistes, a entraîné diverses réactions chez les intellectuels arabes, allant d'un sentiment d'infériorité et d'incomplétude ${ }^{3}$ à l'affirmation

\footnotetext{
${ }^{3}$ L'universitaire tunisien Hamadi Ben Halima s'auto-flagelle en écrivant : «... un bref coup d'œil sur la production littéraire arabe jusqu'au XIX ${ }^{\mathrm{e}}$ siècle nous montre que le poète ou le prosateur manque de souffle et
} 
d'une identité artistique originale. S'inspirant des travaux de Duvigneau, Mohamed Aziza et Chérif Khaznadar rejettent le théâtre à l'occidentale et affirment que les Arabes ont développé leur propre écriture dramatique, différente dans sa forme et son esprit de la tradition occidentale. Ils insistent sur la distinction entre la fête et le thêâtre : la fête réalise l'unité organique de l'espace sacré et de l'espace profane en reliant l'officiant aux spectateursacteurs dans une aire de participation et d'intégration, alors que le théâtre, caractérisé par son caractère symbolique et distanciateur, imite l'existence agissante, mais sans agir lui-même ; le monde arabe connaît la fête, mais pas le théâtre (Aziza, $1975: 7-9$ ).

Khaznadar en tire des conclusions et des recommandations pour le développement futur de la dramaturgie arabe :

L'Occident, après avoir dépassé le stade de la fête et être passé par toutes ces transformations successives, recherche aujourd'hui le moyen de revenir à cette forme originale. Paradoxalement, ces formes qui existent (encore vivantes bien qu'éparses) dans le monde arabe, l'intellectuel arabe les rejette afin de s'engager dans le long cheminement de l'Occident [...] (Tomiche, 1969 : 66).

Il s'agit donc de résister au théâtre à l'occidentale qui ne sera jamais le théâtre arabe, mais uniquement une imitation plus ou moins réussie d'une forme étrangère, n'ayant aucune assise populaire, fruit du labeur et de l'intérêt d'une classe privilégiée souffrant d'un complexe vis-à-vis de la culture des peuples qui l'ont colonisée (Tomiche, 1969 : 59). Et Khaznadar d'appeler au retour à un théâtre participatif de plein air, art total mêlant diverses expressions artistiques.

Aziza va encore plus (trop ?) loin : le théâtre est le fruit de la névrose occidentale, névrose dont l'homme arabo-musulman traditionnel était indemne, car il vivait « l'expérience d'une intégration sans heurt qui résorbe les situations conflictuelles et anomiques et permet, sur le plan du spectacle, qu'une société se donne d'elle-même la pratique harmonieuse de la fête et non celle déchirante du théâtre » (Aziza, 1975 : 14). La colonisation est venue mettre un terme à cette situation harmonieuse et y a introduit le conflit et la frustration, tout en créant la nécessité de nouveaux modes d'expression. $\mathrm{Si}$, dans le monde arabe, le théâtre était né d'une mutation interne des formes traditionnelles du spectacle, fond et forme auraient $\mathrm{pu}$ correspondre, mais le modèle occidental était trop présent, et il s'est imposé au détriment des formes traditionnelles sans pour autant susciter l'intérêt des masses (Aziza, $1975: 25$ ). 
Ahmed Cheniki s'insurge également contre la propension à vouloir faire correspondre les formes artistiques des pays anciennement colonisés avec celles de l'Europe, ce qui mène à établir des rapprochements abusifs. Il refuse d'appeler les formes dites traditionnelles «structures pré-théâtrales », ce qui laisse supposer que nous sommes en présence de formes primaires appelées inéluctablement à donner naissance au phénomène théâtral. Or il s’agit de formes parfaites et achevées, qui obéissent à des normes bien précises (Cheniki, 2002, 2005).

Force est de constater que la question d'une théâtralité indigène et de la définition $d u$ théâtre en milieu arabe achoppe sur des présupposés idéologiques refusant avec véhémence toute ressemblance avec un élément de la culture occidentale, ressemblance automatiquement considérée comme une tare. Prenant le contrepied de la condescendance d'un certain orientalisme, les intellectuels maghrébins lui rendent donc, d'une certaine manière et avec un certain retard, la monnaie de sa pièce.

\section{Les débuts du théâtre « à l'occidentale » en Syrie et en Égypte (XIX ${ }^{\mathrm{e}}$ siècle- 1920)}

Les premiers contacts avec le théâtre européen résultèrent des expériences des voyageurs arabes qui décrivirent l'aspect des salles de théâtre, l'intérêt des Occidentaux pour ce type d'art et s'émerveillèrent des capacités éducatives du théâtre. Par ailleurs, les Français arrivés au Caire avec Bonaparte en 1798 organisèrent des représentations dès la première année d'occupation, et la première salle fut inaugurée en 1800. Après leur départ en 1801, les compagnies théâtrales étrangères continuèrent à se produire au Caire et à Alexandrie, grâce à l'appui de Muhammad 'Ali et de ses successeurs. Plusieurs nouvelles salles virent le jour, tandis que des troupes amateurs et des écoles religieuses organisaient des spectacles, opéras et mélodrames auxquels assistaient, aux côtés des étrangers, les élites locales. En Syrie, c'est surtout dans le milieu des missions chrétiennes que l'activité théâtrale se développa à Damas ou Beyrouth (Hallâq-Toelle, 2007 : 155-158).

La terminologie est assez hésitante à ses débuts : al-Maknâsi traduit théâtre par dâr («maison», «demeure ») en tant que lieu et la $b$ (« jeu») en tant qu'activité, tandis que d'autres préfèrent translittérer les mots français, arabes ou italiens. Le mot masrah, employé aujourd'hui dans les deux sens de «théâtre » (lieu et performance), est un néologisme de la fin du XIX ${ }^{\mathrm{e}}$ siècle qui ne s'affirme définitivement que dans les années 1950 (Hallâq-Toelle, $2007: 158)$.

Le théâtre arabe «à l'occidentale » naît à Beyrouth grâce aux efforts de Mârûn alNaqqâsh. Sa première pièce L'Avare (al-Bakhîl, 1847-1848) est une adaptation libre de la 
pièce de Molière, «arabisée », récitée en arabe littéraire en vers et prose rimée et mise en musique. Le succès qu'elle rencontre l'encourage à en monter d'autres dont certaines sont des adaptations (Le Cheikh ignorant, 1949), et d'autres des créations originales comme Abu alHasan le simplet ou Harun al-Rashid (1849-50), adapté d'un conte des Mille et une Nuits, ou L'Envieux insolent (1851), un drame social dont les héros affirment une liberté nouvelle et très moderne (Hallâq-Toelle, 2007 : 159-161; Allen, 2000 : 196).

À Damas, Abû Khalîl al-Qabbâni monte des pièces comiques inspirées de la littérature populaire et agrémentées de danse, chant et musique. La première d'entre elles, L'Ingrat (Nâkir al-jamîl), est présentée en 1865 dans la cour de la maison familiale. Après des débuts prometteurs, Qabbânî est contraint de quitter Damas chassé par les religieux conservateurs qui considèrent le théâtre comme une innovation satanique. Il se rend en Égypte en 1884, puis à Istanbul et participe à un festival à Chicago, avant de rentrer à Damas en 1900 et d'abandonner la profession pour des raisons mystérieuses. Il a monté un très grand nombre de spectacles et écrit une quinzaine de pièces dont la moitié nous sont parvenues. Le théâtre se répand également à Alep dans les cercles maronites (Yûsuf Ni 'mat Allâh Jadd), et dans les villes palestiniennes sous protectorat britannique (Istîfân Jûzîf Sâlim, Jâmil Habîb al-Bahrî, Salîbâ al-Jawzî) (Hallâq-Toelle, 2007 :162-165).

Le pionnier du théâtre égyptien est Ya'qûb b. Râfâ'îl Sannû' auquel le réformiste Jamâl al-dîn al-Afghânî aurait conseillé de créer un théâtre en langue vernaculaire pour éveiller la conscience du peuple. Il fonda la première compagnie théâtrale égyptienne en 1870 et jouit d'un grand succès populaire. Son engagement politique aux côtés des nationalistes lui valut la disgrâce auprès du Khédive, à la suite de quoi il fut contraint d'abandonner le théâtre en 1872, et l'Égypte en 1978. Il écrivit une trentaine de pièces, mais nous n'avons conservé que le texte de sept d'entre elles, en dialecte égyptien, critiquant de manière acerbe les nouvelles élites de la société égyptienne et la corruption de la classe politique. Son œuvre nous offre toute une série de portraits savoureux : la nouvelle bourgeoisie, l'aristocratie outrageusement occidentalisée, les spéculateurs en bourse, le médecin de formation occidentale aux prises avec les guérisseurs, le fumeur de haschich... (Hallâq-Toelle, 2007 : 165-169 ; Allen, $2000: 198$ ).

Muhammad 'Uthman Jalâl (m. 1894) est une autre figure importante du thêatre égyptien. Il adapta plusieurs pièces de Molière, dont Tartuffe sous le titre d'al-Shaykh Matluf (publié en 1873 mais joué en 1912 seulement), en les transposant dans la société musulmane égyptienne et en utilisant le dialecte égyptien et le genre poétique populaire du zajal. Salîm alNaqqâsh joua en Égypte, de 1875 à 1878, des tragédies de Corneille, de Racine et de Casimir 
Delavigne, faisant ainsi découvrir la tragédie aux Arabes. L'occupation de l'Égypte par l'Angleterre en 1882 éveilla le sentiment national et favorisa la naissance d'un théâtre didactique et historique, qui faisait vivre le glorieux passé arabe ou analysait les raisons du déclin du monde musulman ('Abd Allah al-Nadîm, Mustafâ Kâmil, Farah Antûn, Ibrâhîm Ramzî).

Les premiers comédiens arabes étaient des amateurs, choisis au sein des familles des metteurs en scène ; il y avait peu de femmes et les rares actrices étaient juives ou chrétiennes. Les troupes professionnelles s'appuyaient sur la notoriété d'acteurs-chanteurs comme Yûsuf al-Hayyât (m. 1900), Sulaymân al-Qirdâhî (m. 1909), Salâma Hijâzî (m. 1917), Layla. Le public était issu des élites en Syrie et au Liban, et s'élargissait à la bourgeoisie en Égypte. Les pionniers du théâtre arabe mettaient en avant sa dimension didactique : ils souhaitaient en faire un instrument de la réforme sociale et politique contre les Ottomans, puis les Britanniques (Hallâq-Toelle, 2007 : 169-176 ; Badawi in Jayyusi-Allen, 1995 : 1-2).

$\mathrm{Au}$ début $\mathrm{du} \mathrm{XX}^{\mathrm{e}}$ siècle, de nombreuses troupes se formèrent et se spécialisèrent dans des registres divers : certaines jouèrent exclusivement des pièces lyriques (Salâma Hijâzî, Munîra al-Mahdiyya), d'autres des comédies et des vaudevilles (Najîb al-Rîhânî, 'Alî alKassâr), la tragédie (Georges Abyad), le drame (Georges Abyad, 'Abd al-Rahmân Rushdî, Fâtima Rushdî), le mélodrame et le grand guignol (Yûsuf Wahbî). Ces troupes effectuèrent des tournées dans les pays arabes où elles obtinrent un succès considérable. La Première Guerre mondiale et la crise du coton donnèrent un coup d'arrêt à la recherche théâtrale, alors supplantée par les comédies caricaturales.

Dans les autres régions du monde arabe, le théâtre apparut suite au passage de troupes égyptiennes itinérantes : une troupe visita Tunis en 1908, et vers 1932, il existait déjà quatre troupes locales ; à la suite d'une tournée au Maroc en 1923, une compagnie fut créée l'année suivante ; le même phénomène se produisit en 1926 en Iraq. Cependant plusieurs décennies furent nécessaires à la maturation d'un théâtre autochtone dans ces différentes contrées.

\section{L'essor du théâtre en Égypte}

\section{A. Expérimentations et créativité}

Le début $\mathrm{du} \mathrm{XX}^{\mathrm{e}}$ siècle est marqué par l'apparition d'une nouvelle génération d'auteurs. Farah Antûn (m. 1922) écrivit la première pièce sociale en 1913, Nouvelle et Ancienne Égypte, puis une pièce historique examinant les Croisades d'un point de vue arabe, Salâh al-din et le Royaume de Jérusalem, en 1915. Cette pièce était un appel à la lutte pour la 
libération sous couvert d'histoire, et les autorités britanniques ne s'y trompèrent d'ailleurs pas : elle fut interdite, puis profondément modifiée. Entre les deux guerres, 'Abbâs Allam (1889-1949) composa une série de pièces consacrées à des problèmes sociaux et familiaux, comme Secrets de Palais (1915). Muhammad Taymûr (m. 1921) s’intéressait également à la famille, aux conflits de génération et aux rapports maritaux dans L'Oiseau en cage (1918) ou 'Abd al-Sattâr Efendi (1918). L'Abîme (1921) est consacré à la description d'une addiction, et La Bonne Dizaine (1918) dénonce la politique des gouverneurs turcs en Égypte. Ibrâhîm Ramzî (m. 1949) cultivait à la fois le théâtre historique (Les Héros de Mansura) et social (Un Cri d'enfant). On attribue au poète Ahmad Shawqî la première pièce arabe versifiée : Ali Bey Le Grand (1893, réécrite en 1921) aborde un épisode de la période mamelouke. Il écrivit ensuite, entre 1926 et 1932, de nombreuses tragédies romantiques en vers (La Chute de Cléopâtre, Cambyse, Majnun et Leyla, 'Antara, La Princesse d'Andalousie) et deux comédies (Dame Hoda et L'Avare) (WECT, 1999, IV, 82). Ces pièces versifiées connurent le succès grâce à leur association avec la musique et le chant. Enfin, il existait un théâtre, souvent symboliste et idéaliste, voire philosophique, apparu dans les années 1920 et qualifié de « théâtre à lire ». Un des premiers textes du genre est Iram aux colonnes (1921) de Jibrân Khalîl Jibrân (m. 1931).

Le gouvernement égyptien inaugura en 1930 le premier Institut d'art dramatique qui, cependant, ne fonctionna correctement qu'à partir de 1944. En 1935, il institua également une compagnie nationale comprenant quinze acteurs et huit actrices. Cette troupe, placée sous la direction de Khalîl Mutrân, jouait, en arabe littéraire, des pièces égyptiennes de haut niveau artistique, ainsi que des chefs-d'œuvre du théâtre classique européen. En 1942, la compagnie se dédoubla pour donner naissance à la Troupe égyptienne pour le Théâtre et la Musique, dirigée par Muhammad Hasan, qui mettait en scène des spectacles en arabe dialectal et des opérettes, activité beaucoup plus fédératrice.

Les réalités linguistiques, en Égypte comme dans bien d'autres pays arabes, ont provoqué une division de la production dramatique entre un théâtre dialectal essentiellement destiné à la scène, et un théâtre en arabe littéraire publié en livre avant d'être éventuellement joué. Ibrâhîm Ramzî écrivit ainsi ses pièces «sérieuses »(historiques ou dramatiques) en arabe littéraire, réservant le dialectal aux comédies sociales : Il est plus facile de sortir du hammam que d'y entrer, présentée en 1915 et publiée en 1924, joue sur le scénario connu du notable de province floué par des citadins. Les pièces sociales de Muhammad Taymûr sont également composées en arabe dialectal. Farah Antûn mélange deux niveaux de langue dans sa pièce, Nouvelle Égypte, Ancienne Égypte tandis que ses pièces historiques sont en arabe 
littéraire, de même que celle de Jurjî Zaydân, Saladin et les ruses des Assassins (1913). Quant à Mahmûd Taymûr, il composa et publia deux versions de ses comédies de mœurs, l'une en dialecte et l'autre en arabe littéraire (Abri n 13, 1941 ; Un Thé, 1942). Ces pièces égratignent la bonne société avec humour et légèreté. L'arabe littéraire est également le véhicule du théâtre poétique (Ahmad Shawqî) (Hallâq-Toelle, 2007 : 479-499 ; Badawi in Jayyusi-Allen, $1995: 3)$.

Tawfî̀ al-Hakîm (1898-1987) est sans doute le dramaturge égyptien le plus connu, eu égard à la longueur de sa carrière (un demi-siècle, des années 1920 aux années 1970) et son exceptionnelle productivité (plus de 80 œuvres). Encore étudiant, il composa, d'abord de façon anonyme, des pièces musicales ou des satires de la vie politique ou sociale locale. Lorsque son identité fut révélée, son père scandalisé l'envoya en Europe afin qu'il y termine ses études de droit. Il y découvrit un théâtre considéré comme une forme littéraire sérieuse, et non comme un simple divertissement. Revenu en Égypte en 1928, il entreprit de rénover le théâtre égyptien et d'en faire une forme littéraire reconnue.

Sa première pièce, Les Gens de la Grotte (1933), est une reprise du conte des Sept Dormants d'Éphèse, relatée dans la sourate 80 du Coran, où il exploite les thèmes de la naissance à un autre monde et du retour au passé. Il écrivit ensuite Schéhérazade (1934), dont l'héroïne s'avère être la source de vie et de sagesse à laquelle aspire Shahriyâr, guéri de ses pulsions meurtrières. La pièce fut jouée par la Troupe Nationale de Théâtre en 1935 et ne connut pas le succès, car l'absence d'action de ce «théâtre des idées » déconcertait le public. Ce qui n'empêcha pas al-Hakîm de continuer à composer des pièces philosophiques : Barakassa ou le problème du pouvoir (1942), Pygmalion (1942), Salomon le Sage (1943), Le Roi Edipe (1949). Il s'y montre inspiré par le théâtre d'avant-garde européen et par Luigi Pirandello. Parallèlement, il traita des thèmes extrêmement variés dans des registres différents allant de la comédie de mœurs (Le Secret du suicide, 1928 ; Une Balle dans le cœur, 1939) au drame social (Une Vie maudite).

Il écrivit également des pièces courtes qui n'étaient pas destinées à être jouées, mais publiées dans les journaux, et dont la meilleure, Chant de mort, décrit l'atmosphère d'un village de Haute Égypte, où une famille attend le retour d'un fils étudiant dans l'espoir qu'il règle une dette de sang. En 1950, il fit paraître sous le titre de Pièces sociales un ensemble de vingt-et-une pièces parues dans Akhbâr al-Yawm entre 1945 et 1950 : leurs dialogues vivants et la fine analyse des problèmes sociaux du pays au lendemain de la Seconde Guerre mondiale leur assurèrent un grand succès ; il y fustigeait notamment la corruption politique jusqu'aux 
plus hauts degrés de l'État, le népotisme, les abus de pouvoir, les méfaits du capitalisme sauvage et le matérialisme prévalent.

La Révolution de 1952 marqua un tournant dans son œuvre : il y concilia harmonieusement le théâtre «d'idées » et le théâtre populaire, et en conséquence beaucoup des pièces écrites dans les années 1950 et 1960 furent portées à la scène. Les Mains douces (1954) sont un plaidoyer pour la réconciliation nationale et effleurent la question de la place des intellectuels dans la nouvelle société. Sa Majesté (1955) attaque violemment la corruption de l'ancien régime. Shams al-Nahar (1964) est une pièce brechtienne glorifiant le travail, l'égalité et la justice. Le Sultan perplexe (1960) insiste sur la nécessité pour les puissants de respecter les lois. Il expérimenta aussi d'autres moyens de s'exprimer que l'arabe littéral, inventant un langage intermédiaire entre le dialectal et la langue savante dans Le Contrat (1956) qui aborde le thème de l'exploitation des paysans par les propriétaires terriens.

À la suite d'un séjour en France en tant que représentant de l'Égypte à l'UNESCO, il s'intéressa au théâtre de l'Absurde et écrivit en 1962 Ô toi qui grimpes à l'arbre, pièce «nonlogique » (lâ ma'qûll): un vieux couple y poursuit deux monologues parallèles, chaque personnage étant sourd à l'autre. De la nourriture pour toutes les bouches (1963) traite de la science et de la responsabilité. Déçu par la Révolution, il composa des pièces grinçantes et sombres où la critique se dissimule sous la parabole et l'allégorie : Le Destin du cafard (1966) se compose de deux pièces juxtaposées, une satire du monde politique et la description d'une société gouvernée par un cafard [figure 123] ; Un Voyage en train (1964) et Chaque Chose à sa place (1967) relèvent de la même inspiration. Sa dernière pièce, intitulée Le Monde est une farce, est une fantaisie pastorale. Ses thèmes de prédilection sont la modernisation du monde arabe, les problèmes liés à l'exercice du pouvoir, la dualité de l'esprit et de la matière (Badawi in Jayyusi-Allen, 1995 : 3-5 ; Allen, 2000 : 201-204). 


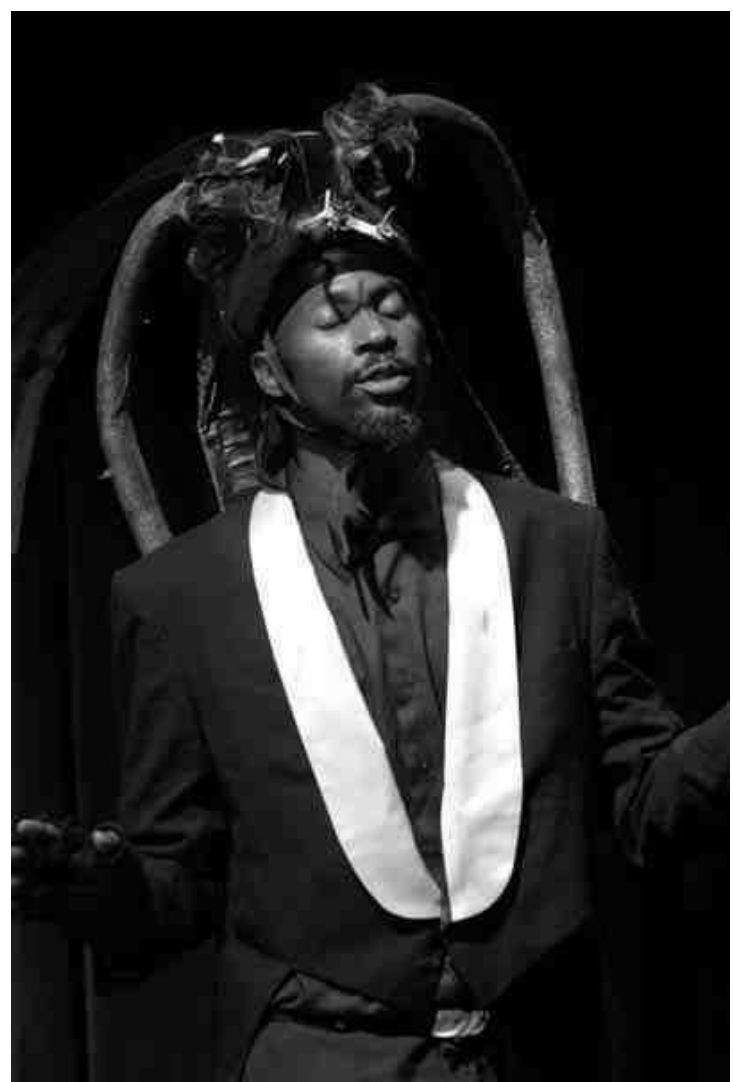

Figure 123 : Le Destin d'un cafard de Tawfiq al-Hakim, joué par le Sanctuary Theatre en 2007 dans le cadre du Fringe Festival, photo anonyme, http://sanctuarytheatredc.org/?page id=55

\section{B. Engagement socio-politique et diversification des formes}

Une nouvelle génération de dramaturges apparut dans les années 1940 dans le sillage d'al-Hakîm. Le poète 'Azîz Abâza composa des pièces versifiées traitant de l'histoire des Arabes, Qays et Lubna (1943), al-Nâsir (1949), Crépuscule (1952), Caravane de lumière (1959), ainsi que deux pièces plus contemporaines, Feuilles d'automne (1957) et Zahra (1969). Le nouvelliste Mahmûd Taymûr (1894-1973) cultiva divers registres : pièces historiques (Éternelle Ève, Du Vin aujourd'hui, Tariq d'Andalousie), basées sur la légende (Épouse du Nil, Plus Malin que Satan), ou sociales (Abri $n^{\circ} 13$, Bombes, Mensonge après mensonge, Les Faussaires). Son écriture élégante, sa sensibilité théâtrale et ses personnages à la psychologie fouillée en font un excellent dramaturge. Yéménite de naissance mais Égyptien d'adoption, 'Alî Ahmad Bakâthîr (1910-1969) se tourna résolument vers les questions politiques : Dieu d'Israël, Le Peuple élu, L'Ongle de Juhâ traitent ouvertement du conflit arabo-israélien, du sionisme et de l'impérialisme, tandis que ses pièces historiques sont une réponse à des événements contemporains (Hârut et Mârut, Al-Hakim bi amr Allâh). Il a 
également écrit des comédies sociales (Chats et souris, Madame Julfadan). La quasi-totalité de son œuvre est en arabe classique (WECT, 1999, IV, 82).

Après la Révolution, le Ministère de la Culture appuya financièrement de nombreux théâtres et compagnies, des directeurs formés en Europe revinrent en Égypte, et la critique théâtrale se développa, notamment grâce à la nouvelle revue Al-Masrah et à la publication de séries de textes théâtraux égyptiens ou de chefs-d'œuvre mondiaux en traduction. Les jeunes auteurs, initialement très enthousiastes, reprirent les slogans nationalistes et socialistes, et utilisèrent volontiers le dialectal. Le théâtre devint une tribune politique, et le resta lorsque le totalitarisme et la censure s'installèrent peu à peu. Le monde du théâtre resta cependant ouvert aux influences occidentales, appréciant tout particulièrement Eugène Ionesco, Samuel Beckett, John Osborne, Bertold Brecht. L’intérêt pour le «théâtre total » ou «le théâtre en rond », et le nationalisme ambiant incitèrent les dramaturges et les théoriciens à tenter de créer un théâtre spécifiquement arabe et égyptien, tandis que l'on s'interrogeait sur la nature du théâtre et sur son rôle au sein de la société. On chercha à s'inspirer de formes traditionnelles comme le théâtre d'ombres, al-samir, veillée villageoise animée par le chant, la danse et les conteurs, ou la maqâma, forme littéraire arabe médiévale en prose rimée, agrémentée de poésie, narrant les aventures d'un sympathique vagabond.

'Abd al-Rahmân al-Sharqawî (m. 1988) composa une pièce en vers libres traitant de la lutte du peuple algérien contre le colonialisme français, La Tragédie de Jamila (1962). Ses autres pièces, dont la meilleure est Mahran le chevaleresque, abordent les réalités de l'Égypte contemporaine, la lutte arabe, l'injustice et la corruption. Le théâtre de Najîb Surûr (m. 1978) est tout aussi politique, qu'il fustige le féodalisme (Yassin et Bahiya, 1964), dénonce le colonialisme ( $\hat{O}$ Nuit, ô lune, 1968), s'interroge sur les causes de la défaite de 1967 et la faillite de l'intelligentsia ( $L$ 'CEil du soleil, 1973), ou encore la corruption politique (Où puis-je trouver les bonnes personnes ?, 1984) dans une langue familière, colorée et imagée. Salâh 'Abd al-Sabûr (m. 1981) innove en composant des pièces teintées de réalisme symbolique, qui décrivent la difficulté des intellectuels à s'investir dans des actions concrètes (La Tragédie de Hallâj, Leyla et Majnun, 1970, Le Voyageur nocturne) (WECT, 1999, IV, 84).

Yûsuf Idrîs décrit la lutte des paysans contre le féodalisme dans Le Roi du coton (1957), et la résistance à Port-Saïd en 1956 dans Le Moment critique (1958). Dans Les Claquettes (1964), il se base sur la tradition folklorique égyptienne (samir, Juha) et s'efforce de briser les frontières entre acteurs et public : la pièce commence avec le discours de l'auteur habillé de manière ridicule et vite interrompu par l'intrusion de Farfur, un domestique à la recherche d'un maître ; au fur et à mesure que le rôle de l'auteur s'amenuise, la relation 
tumultueuse entre le maître et le domestique prend de l'importance et ne semble même pas pouvoir être réglée par l'inversion des rôles. Les thèmes de l'autorité et de la liberté, de la hiérarchisation de la société, de la corruption du pouvoir et de la désertion du monde par Dieu sont abordés avec un certain humour. Idrîs continue à manier le symbolisme et l'absurde dans ses autres pièces, notamment Les Rayés (1960), où il s'en prend violemment au parti unique de l'ère Naser et Le Troisième Sexe (1971) qui fustige les dérives de la modernité (Badawi in Jayyusi-Allen, 1995 : 6-10).

À côté de ces auteurs en quête de nouvelles formes poétiques, le réalisme connaît une vogue sans précédent. L'œuvre de Nu'mân 'Ashûr (m. 1987) relève de la critique sociale : ses pièces retracent l'histoire d'une famille égyptienne de 1940 à 1980 et montrent comment évoluent les valeurs et rapports sociaux. Les Gens d'en bas, Les Gens d'en haut, La Famille Dughri (1963) décrivent, de façon réaliste et en dialectal, les interactions d'une poignée de familles partageant le même immeuble, en particulier le clash entre le matérialisme de la vieille génération et l'idéalisme de la jeunesse. L'auteur s'intéresse beaucoup à la jeune génération en perte de repères (Les Pays d'outre-part, 1967 ; La Tour, 1978 ; Le Jouet, 1983).

Râshid Rushdî écrit d'abord des pièces psychologiques (Le Papillon, Voyage derrière le mur, La Douceur du passé, 1967, La Lumière des ténèbres, 1971) avant de se politiser avec Regarde, bon sang (1966) qui montre le despotisme mamelouke, et Ma Chère Patrie (1968) qui analyse la passivité des populations face à l'invasion. Sa'd al-dîn Wahbah (né en 1925) s'intéresse tout particulièrement à la question du pouvoir et des relations entre le gouvernant et ses administrés : Le Pont aux moustiques (1964) montre que l'oppression avait rendu inévitable la Révolution de 1952 ; La Route vers la sécurité (1965) aborde divers problèmes sociaux comme l'opportunisme et la corruption qui gangrènent le nouveau régime socialiste ; Les Ongles (1969) revient sur le déroute de 1967 et ses conséquences ; Le Professeur (1969) se penche sur le totalitarisme (WECT, 1999, IV, 84-85).

Mikhâ'îl Rûman (1927-1973) analyse finement les contradictions de l'intelligentsia égyptienne, insatisfaite mais incapable d'influer sur la société, à travers les mésaventures de Hamdi, un homme frustré dont la révolte reste stérile (La Fumée, 1962, Le Nouveau Venu, 1966). Emprunté et loué (1967) et le Rédacteur de réclamations (1968) se penchent sur la bureaucratie, la corruption et les faux-semblants. La Nuit où on a tué Che Guevara (1969) examine la confrontation entre le néocolonialisme et les mouvements de libération. Mahmûd Diyâb (m. 1983) s'intéresse avant tout à la vie des villages. Ainsi La Tempête (1967) décrit le trouble entraîné dans un village par le retour d'un homme accusé à tort d'un crime, emprisonné pendant vingt ans et dont on craint la vengeance. 
'Alî Salîm (né en 1936) excelle dans la satire, fustigeant les disfonctionnements politiques (corruption, despotisme) et les lourdeurs de la bureaucratie étatique, tantôt dans un registre fantastique (Peuple du Huitième Ciel, 1966, Le Voyage de Noé, 1975), tantôt de façon réaliste (La Comédie d'Edipe: Vous avez tué la bête, 1970 ; Master en management de peuples, 1979). Dans La Comédie d'Edipe, un simple citoyen de Thèbes, Edipe, se porte volontaire pour résoudre l'énigme proposée par le Sphinx. Il réussit, négocie avec la Bête, est couronné roi, et épouse la reine Jocaste. Tandis que la propagande officielle le transforme en héros crédité de la mise à mort de la Bête, Jocaste, insatisfaite, complote contre lui. L'apparition d'une seconde Bête lui offre l'occasion de se débarasser de lui : le pauvre CEdipe est poussé dans la gueule du loup. De nombreuses allusions à la situation politique à la veille de la disparition de Naser font de cette pièce une illustration de la Révolution égyptienne.

'Abd al-'Azîz Hammûda (né en 1938) expérimente au niveau de la forme en s'inspirant de la tradition des conteurs et des légendes pharaoniques, pour se pencher sur le pouvoir tel qu'il est vécu par le peuple dans Les Otages (1982) ou La Dernière Nuit du colonel (1983). Fathiya al-'Asal (née en 1933) est une des rares femmes dramaturges ; elle écrit pour la télévision et examine la condition féminine, caractérisée par la soumission et la peur (Femmes sans masques, 1982 ; La Prison des femmes, 1993) (WECT, 1999, IV, 85-86).

Quelques auteurs continuent à écrire en arabe littéral de façon à toucher un public panarabe. L'un d'entre eux est Alfred Faraj (né en 1929) avec des pièces comme Le Barbier de Bagdad (1963), Salomon d'Alep (1965), Soldats et voleurs (1966), al-Zir Salim (1967), Ali Janah de Tabriz (1969) et Messages du Juge de Séville (1975), qui s'appuient sur des éléments du patrimoine littéraire arabe pour construire des allégories de la situation politique et sociale contemporaine. Ainsi, la Chute de Pharaon (1957) se réfère à la chute de la royauté en 1952. Influencé par Ionesco et Beckett, Mustafâ Bahjat mélange rêve et réalité et montre l'absurdité d'une vie dirigée par des forces occultes dans Le Procès de la famille Dabash (1971) et Zomorroda (1984).

L'instabilité sociale à partir des années 1970 et le poids de la censure ont peu à peu changé la donne : les pièces légères remplacent le théâtre plus ambitieux, la presse se tait, les jeunes auteurs tentent de développer le Happening et le Living Theatre qui tient plus du meeting politique que de l'art, et impose l'optimisme comme devoir national. On recherche de nouveaux modèles. Dans sa trilogie Un Brave Homme à travers trois contes, Diyâb imite Ionesco et son théâtre de l'Absurde, tandis que dans La Tempête, Les Vauriens, Les Journées de récolte, il s'inspire du samir. Le doute atteint également les héros : le Saladin de Diyâb n'a plus rien d'un grand homme, tandis qu'Antara, le héros de la légende populaire, n'est plus, 
chez Yusrî al-Jindî, qu'un vaincu pathétique, incapable de sauver sa tribu. Le théâtre devient cependant de plus en plus commercial dans les années 1990, en dépit de la création d'un festival de théâtre expérimental au Caire en 1988, et de la Maison de l'Opéra en 1996 (Badawi in Jayyusi-Allen, 1995, 7-10).

\section{Le théâtre au Moyen-Orient}

\section{A. Syrie : un théâtre politique}

En Syrie et au Liban, où l'activité théâtrale avait commencé relativement tôt avant d'être stoppée dans son élan par la censure et le départ massif des intellectuels vers l'Égypte, nombre de critiques considèrent que les réels débuts du théâtre dans la région coïncident avec la création des théâtres nationaux dans les années 1960 à 1970.

En réalité, des troupes d'amateurs furent actives en Syrie dès le tout début du $\mathrm{XX}^{\mathrm{e}}$ siècle, en dépit des pressions religieuses, et les autorités françaises les encouragèrent pendant la période coloniale de 1920 à 1940, contribuant, à leur corps défendant, à l'élaboration d'une culture et d'une littérature nationales. Les clubs artistiques et littéraires se multiplièrent rapidement et adaptèrent des pièces du répertoire international aux réalités locales. Le premier acteur professionnel, 'Abd al-Latîf Fathî (1916-1985), joua un rôle important dans cette « syrianisation » du théâtre ; il devint d'ailleurs le premier directeur du Théâtre Populaire (alMasrah al-sha 'b̂̂), en activité de 1964 à 1970, date à laquelle il fusionna avec le Théâtre National (al-Masrah al-Qawmî) créé en 1960. À partir des années 1960, le Ministère de la Culture encouragea l'écriture dramatique en organisant des concours.

L’auteur syrien le plus réputé, Sa‘dallâh Wannûs (1940-1997), dramaturge, critique théâtral et directeur, combine thèmes classiques et techniques modernes. Il se fit connaître par Soirée du 5 juin (1968), une pièce cinglante qui dénonçait les attitudes de la société arabe confrontée à la défaite face à Israël en juin 1967. Dans cette pièce, des acteurs sont censés représenter la version officielle des événements, mais ils sont constamment dérangés par des spectateurs qui montent sur scène pour exposer leur version des faits ; au fur et à mesure que la réalité de la défaite se fait jour, les officiels au premier rang s'agitent, avant d'interrompre la pièce et d'arrêter acteurs et public. Wannûs avait auparavant écrit une série de pièces en un seul acte à la manière du théâtre d'idées d'al-Hakîm, mais en utilisant les techniques du théâtre de marionnettes : Le Cadavre sur le pavement montre de manière surréaliste comment le pouvoir prend systématiquement le parti des riches et des notables contre les pauvres ; 
L'Éléphant, ô roi du monde ! est une parabole de la terreur que fait régner sur son peuple un gouvernant tyrannique [figure 124].

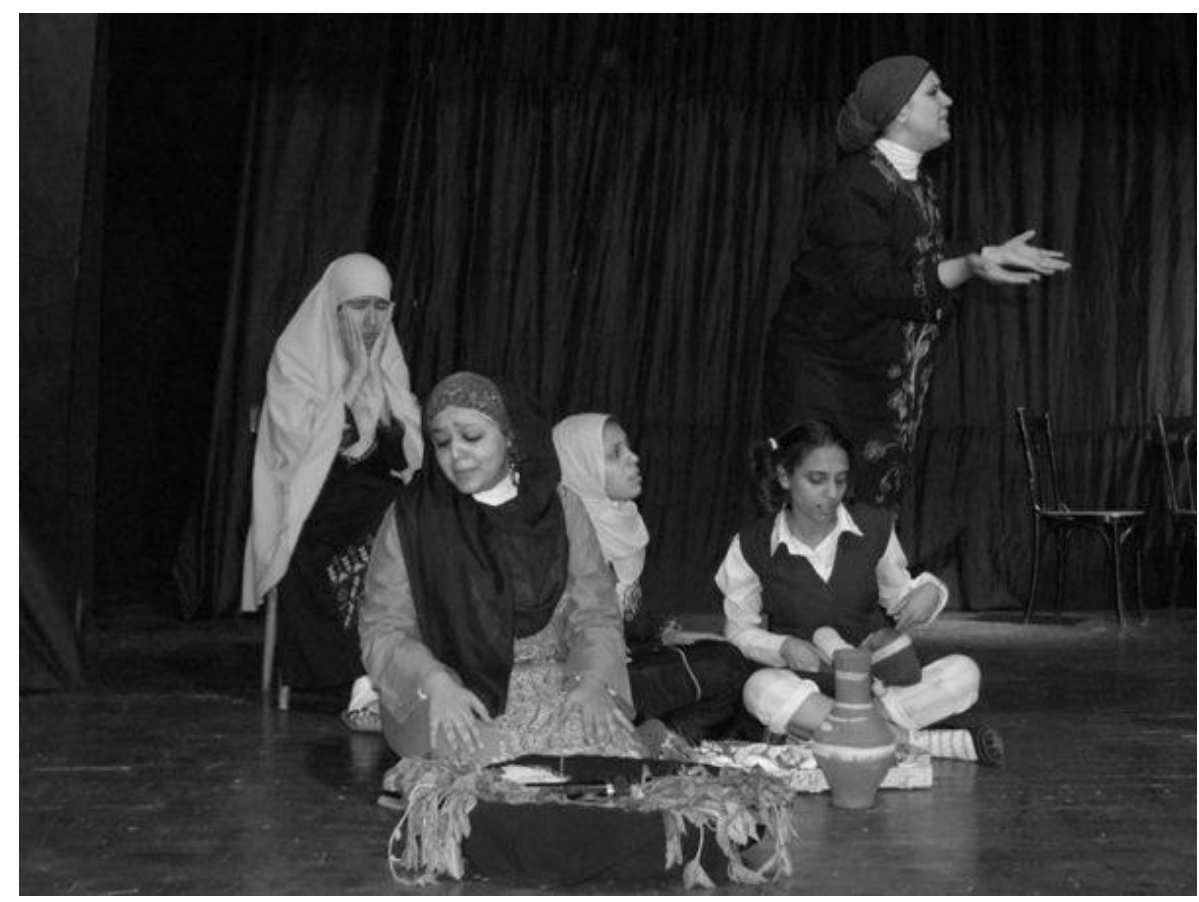

Figure 124 : L’Éléphant, ô Seigneur du monde! de Sa'dallâh Wannûs, joué le 12/05/2011 à l'Université du Caire, théâtre de la Faculté de droit.

URL: http://weekly.ahram.org.eg/2011/1049/cu2.htm

Influencé par Piscator et Brecht, Wannûs milite pour la politisation du théâtre censé éduquer l'audience, et veille à l'interaction avec le public, recourant dans ce but à l'adoption de techniques empruntées aux spectacles traditionnels comme à l'Occident. C'est pourquoi il construit sa pièce Les Aventures de la tête du mamlouk Jabir (1969) comme un canevas pour la représentation, conseillant de traduire le texte dans la langue de la région et d'agrémenter la performance de musique locale. Une Soirée avec Abu Khalil al-Qabbani (1972) prend la forme originale d'une pièce dans la pièce. Le Roi c'est le roi (1977) s'inspire d'un conte des Mille et Une Nuits.

Toutes ces pièces portent un message politique révolutionnaire. Dans Les Aventures de la tête du mamlouk Jabir, l'action se déroule dans un café où des clients prennent un verre tout en écoutant de la musique à la radio. Ils attendent la venue d'un conteur traditionnel (hakawâtî), mais lorsque celui-ci se présente, au lieu de leur raconter les victoires de Baïbars contre les croisés, il narre une querelle entre un calife abbasside et son vizir, lequel envoie Jabir porter un message secret écrit sur sa tête au roi de Perse. Une fois le message délivré, 
Jabir est mis à mort au lieu d'être récompensé : le message conseillait au roi perse d'envahir Bagdad et de renverser le calife, mais aussi de tuer le messager ! Les événements racontés sont représentés par des acteurs, le conteur joue le rôle du chœur, les clients commentent l'action. Les trois plans sont interdépendants, et les allusions aux réalités du monde arabe contemporain nombreuses.

Dans Une Soirée avec Abu Khalil al-Qabbani, trois thématiques se trouvent entrelacées : une pièce d'Al-Qabbânî est jouée par des acteurs, tandis que la carrière d'alQabbânî comme dramaturge et fondateur du théâtre syrien est mise en scène parallèlement, et que Wannûs fournit un commentaire politique à la situation du pouvoir ottoman. Dans Le Roi est le roi, la blague de Hârûn al-Rashîd droguant un marchand pour l'installer une journée sur le trône tourne mal : Abû 'Izza exerce un pouvoir absolu et tyrannique et refuse de rendre le trône à Hârûn al-Rashîd. Au début de la pièce, les grandes lignes de l'action sont présentées par un chœur qui réapparaît aux moments clefs pour commenter les événements, et s'adresse à la fin à l'audience pour lui conseiller de se débarrasser des rois (Badawi in Jayyusi-Allen, 1995 :10-12 ; Allen, 2000 : 209-211).

Les autres dramaturges syriens des années 1970 cultivent généralement un théâtre politisé. Dans Le Derviche cherche la vérité (1970), Mustafâ al-Hallâj décrit l'infortune d'un homme innocent torturé et mis à mort parce qu'il porte le même nom qu'un activiste politique recherché par les services secrets. Mamdûh 'Udwân (né en 1941) commente le monde politique arabe contemporain à travers une action et des personnages basés sur les premiers siècles de l'histoire de l'islam (La Nuit des esclaves, 1977). 'Alî 'Uqla 'Ursân (né en 1940) décrit allégoriquement l'immigration sioniste en Palestine dans Les Étrangers (1974), et les persécutions politiques dans le monde arabe dans Prisonnier $n^{\circ} 95$ (1974).

Dans un registre plus léger et plus satirique, Walîd Ikhlâsî (né en 1935) nous livre une galerie de beaux portraits, sans doute les personnages les mieux définis du théâtre arabe. Un opportuniste parvenu au poste de directeur de cabinet ministériel raconte son ascension jalonnée par l'hypocrisie, l'absence de conscience et d'amour-propre, l'ingéniosité, la flagornerie et les mauvais coups dans Comment grimper sans tomber (1973). La Rivière folle (1976) présente une dame âgée à la forte personnalité, appartenant à la classe supérieure, qui n'arrive pas à accepter l'expropriation dont elle a fait l'objet suite à la réforme agraire, et se réfugie dans un monde imaginaire. Dans Le Chemin (1976), un petit employé de théâtre est amené à remplacer au pied levé un acteur malade et connaît un immense succès comique, mais déchante en découvrant que sa popularité se limite à la scène et que sa fortune est bâtie sur le mensonge et l'hypocrisie ; il chemine entre le bien et le mal avant de se soumettre au 
pouvoir et à la police secrète. Il représente la crise de l'écrivain arabe sous le joug d'un système totalitaire.

Muhammad al-Maghût (né en 1932) s'est fait connaître par sa pièce surréaliste et violente Le Moineau bossu (1968), une fable sur l'oppression politique, et surtout Le Bouffon, qui dévoile l'inanité des rêves et mythes arabes ainsi que la tyrannie et l'absurdité des régimes, à travers la circulation de personnages jadis célèbres et vénérés, entre un passé glorieux et un présent qui les méconnait ou les persécute.

Depuis 1976, un festival annuel a lieu à Bosra dans un amphithéâtre romain. Le festival de Damas, créé en 1969, accueillait les principales créations arabes contemporaines avant d'être relayé à partir de 1988 par le festival Al-Mahabba de Lattaquieh. Des compagnies commerciales ont vu le jour : Firqat Dabâbîs créée en 1973 et dirigé par le dramaturge Ahmad Qanu' (1936-1998), a produit Missiles, Destin et chance ; Firqat Mahmûd Jabr a produit Le Pillage (1977), Un Bain sans eau courante (1979) et Le Maître d'école (1979). Ces pièces en dialectal sont souvent jouées pendant des années. Depuis la fin des années 1980, le théâtre syrien a tendance à stagner et les acteurs se reconvertissent dans les séries télévisées (Badawi in Jayyusi-Allen, $1995: 13-16)$.

\section{B. Liban : avant-gardisme et contestation}

La production théâtrale libanaise devient remarquable dès les années 1940 avec, d'une part, des pièces romantiques comme Le Bien et le mal de 'Abd Allâh Hushayma, ou L'Épouse rebelle de Yûsuf al-Qâdi, d'autre part, des pièces religieuses chrétiennes produites par des clercs à l'exemple de L'Assomption de Yûsuf Bahît ou Sainte Jeanne d'Arc de Yûsuf alHâyik. Les problèmes de société font leur apparition avec de nouveaux personnages : le jeune homme européanisé, la jeune fille émancipée, l'ivrogne, le joueur, l'opportuniste, la mère vertueuse, le citoyen défenseur des traditions, le paysan attaché à sa terre. La ville, réservoir à problèmes sociaux et moraux, s'oppose à la campagne perçue comme la gardienne des valeurs traditionnelles. Ces idées se reflètent dans les pièces de Farîd Mudawwar (C'est étrange, La Revanche), Edwâr al-Dahdâh (Une Tempête dans un village, Les Injustices de la vie), Josef alGharîb (L'Amant distrait). Dans Pères et fils (1916), Mikhâ'îl Nu'aymah décrit les ravages de la corruption et des mariages arrangés dans la communauté chrétienne libanaise, tout en faisant parler certains de ses personnages en dialectal. Le poète Sa'îd 'Aql compose un drame en vers inspiré du mythe grec du rapt d'Europe par Zeus, Qadmus (1944). 
Les années 1960 marquent une véritable révolution du théâtre libanais grâce aux efforts d'Antoine Multaqâ et de Munîr Abû Dibs qui s'inspirent largement des nouvelles tendances du théâtre occidental. Le théâtre est désormais vu comme une performance et une expérience vivante plutôt qu'un genre littéraire. Il s'adresse à un public éduqué, bien au fait des méthodes avant-gardistes du théâtre occidental et à même de comprendre les subtilités voilées des satires politiques. Antoine Multaqâ crée une École de théâtre et une nouvelle troupe, Firqat al-masrah al-hadîth, puis une autre compagnie, le Théâtre en rond libanais, et enfin le Théâtre du Conteur, qui rejette les limites de la scène, les conventions scéniques, le décor, et recourt au modèle du hakawâtî, au folklore et aux symboles pour inciter les spectateurs à exercer leur imagination. Le texte de la pièce devient un simple canevas sur lequel les acteurs improvisent collectivement, avec la collaboration de l'auteur-metteur en scène. Au nombre de ces nouveaux auteurs, il faut citer Abû Dibs (L'Inondation, Jibrân, Jésus, Ombres), et Remon Jbâra (né en 1935, Laissons mourir Desdémone, Aux bons soins de Zakkur). Le poète 'Isâm Mahfûz (né en 1939) a écrit un certain nombre de pièces « littéraires »: L'Arbre de Chine (1963), Le Meurtre (1968), Le Dictateur (1970), Pourquoi ? (1971). La première de ces pièces tient à la fois de Kafka et du théâtre de l'Absurde : un homme y est jugé pour le meurtre d'une femme et condamné à mort, alors que les charges ne sont jamais établies. Il entend une mystérieuse voix qui s'appelle elle-même arbre. Par la force de sa volonté, il se transforme en arbre.

Les auteurs étrangers les plus divers sont traduits par des personnalités telles que 'Unsî al-Hâjj, Adonis, 'Isâm Mahfûz. Le dialectal est fréquemment utilisé et les scripts sont « libanisés », tandis que le théâtre tend à devenir un art «total » intégrant la musique et la danse. Le festival de Baalbeck joue, jusqu'à son interruption en 1977, un rôle essentiel d'ouverture sur les grands courants internationaux, ainsi que les théâtres de langue française (Centre universitaire d'études dramatiques), anglaise (Drama Club de l'Université américaine), et arménienne.

Le traumatisme de la guerre arabo-israélienne de 1967 a inspiré de nombreuses pièces ; la plupart expriment l'amertume et la frustration face à des dirigeants arabes perçus comme incompétents et tyranniques. Le Dictateur critique ouvertement un régime militaire uniquement préoccupé par sa propre survie. Majdalun de Henry Hâmatî orchestre un dialogue violent entre le peuple et le gouvernement, tout en abordant les déchirements entre rebelles, combattants palestiniens, autorité politique et Isräl. Le Clown de Mohammad al-Maghût compare le passé arabe glorieux et l'humiliation présente. La République des animaux (1971) 
de Shakîb Khûrî attaque les régimes militaires arabes, venus au pouvoir pour promouvoir le socialisme, mais foulant aux pieds l'intérêt public (Badawi in Jayyusi-Allen, 1995, 16-17).

Avec la guerre civile de 1975 à 1990, la recherche théâtrale s'interrompit, et seules les comédies légères et les spectacles de variétés survécurent (WECT, 1999, IV, 140-147).

\section{Palestine : le reflet d'un drame humain et politique}

Si les troupes étaient nombreuses en Palestine avant 1948, la création de l'État d'Israël, l'exode de l'intelligentsia vers d'autres pays arabes et la censure ne favorisèrent pas le développement du théâtre. Pourtant le théâtre y était né très tôt : le journaliste Jamîl alBahârî (m. 1929) adapta ou composa seize tragédies en vers (Le Fratricide, 1919 ; Le Prisonnier du palais, 1920 ; Patrie bien aimée, 1923). De nombreuses troupes s'étaient formées à Jaffa, Nazareth, Gaza et Jérusalem dans les années 1920. L’écrivain palestinien le plus prolifique est assurément Nasrî al-Jûzî qui écrivit une centaine de pièces entre 1920 et 1970 (La Vérité suprême, 1927 ; Les Chandelles se consumant, 1930 ; Ma Mère). Son théâtre, didactique et montré essentiellement dans les écoles, est toujours simple et moralisateur. La plupart des pièces antérieures à la Seconde Guerre mondiale étaient écrites en arabe classique et s'adressaient à un public cultivé et prospère.

Le théâtre politique de Mu'în Bisûson (1926-1984) aborde les relations tumultueuses entre la Palestine et Israël à travers l'évocation d'une histoire plus ancienne. La Révolte des Zanj, présentée pour la première fois au Caire en 1970, rapproche le sort des Palestiniens de celui de ces esclaves noirs que furent les Zanj. Samson et Dalila (Le Caire, 1971) raconte le destin d'une famille palestinienne contrainte de s'exiler, puis de se préparer à combattre. Ses autres pièces, Les Oiseaux construisent leurs nids entre les doigts (1973), La Tragédie de Guevara, Le Procès du Livre de Kalila et Dimna, ont pour principal thème la discrimination et l'oppression. Ghasân Kanafânî (1936-1972) décrit l'exil comme une sorte de mort dans La Porte (1964) et Un Pont vers l'éternité. Râshid Abû Shawûr construit ses pièces sur des oppositions entre le présent et le passé, la maison et le camp de réfugiés, la victoire et la défaite, la traîtrise et le patriotisme, la mort et la naissance (Le Rêve palestinien, L'Étranger et le Sultan).

Le théâtre de cette époque est essentiellement idéologique et on y lit clairement la transformation progressive des Palestiniens de fugitifs chassés de leur patrie en combattants pour la liberté, de vaincus en révolutionnaires. Le théâtre palestinien en Syrie, particulièrement actif, était sponsorisé par l'OLP (Un Peuple qui ne mourra pas, La Voie). 
Samîh al-Qâsim (né en 1939) y écrivit Institut de la Folie Nationale (1977), un réquisitoire contre la politique israélienne. La Troupe des Ballons (1971-1974) s'établit à Jérusalem et monta Qarqash d'al-Qâsim, Une Tranche de vie, L'Obscurité, Prévisions météo. Toutes ses pièces critiquent des aspects de la société palestinienne : la mise à l'écart des femmes, l'impossibilité de faire front commun, l'exploitation des travailleurs, le pouvoir militaire. La troupe Hakawâtî (1977-1993) montait des pièces écrites collectivement et faisant une large part à l'improvisation et à l'expérimentation, et se produisait sur les places de village plutôt que dans des salles régulières. Le Village de Shamma raconte l'histoire d'un jeune Palestinien qui part étudier à l'étranger et qui, à son retour, ne parvient pas à retrouver son village, littéralement rayé de la carte. D’autres troupes comme Les Ongles, al-Kashkul, Épis de blé, eurent une existence assez éphémère. L'isolation, l'occupation israélienne et le conservatisme religieux sont loin de faciliter les choses, et pourtant le théâtre palestinien semble actuellement renaître de ses cendres avec, notamment, l'apparition d'échanges avec la scène israélienne, la création d'une Union théâtrale palestinienne et l'émergence de nouvelles troupes comme Ashtar, inspirée du théâtre de l'opprimé d'Augusto Boal [figure 125] (WECT, 1999, IV, 187-196 ; Badawi in Jayyusi-Allen, 1995 : 17-18).

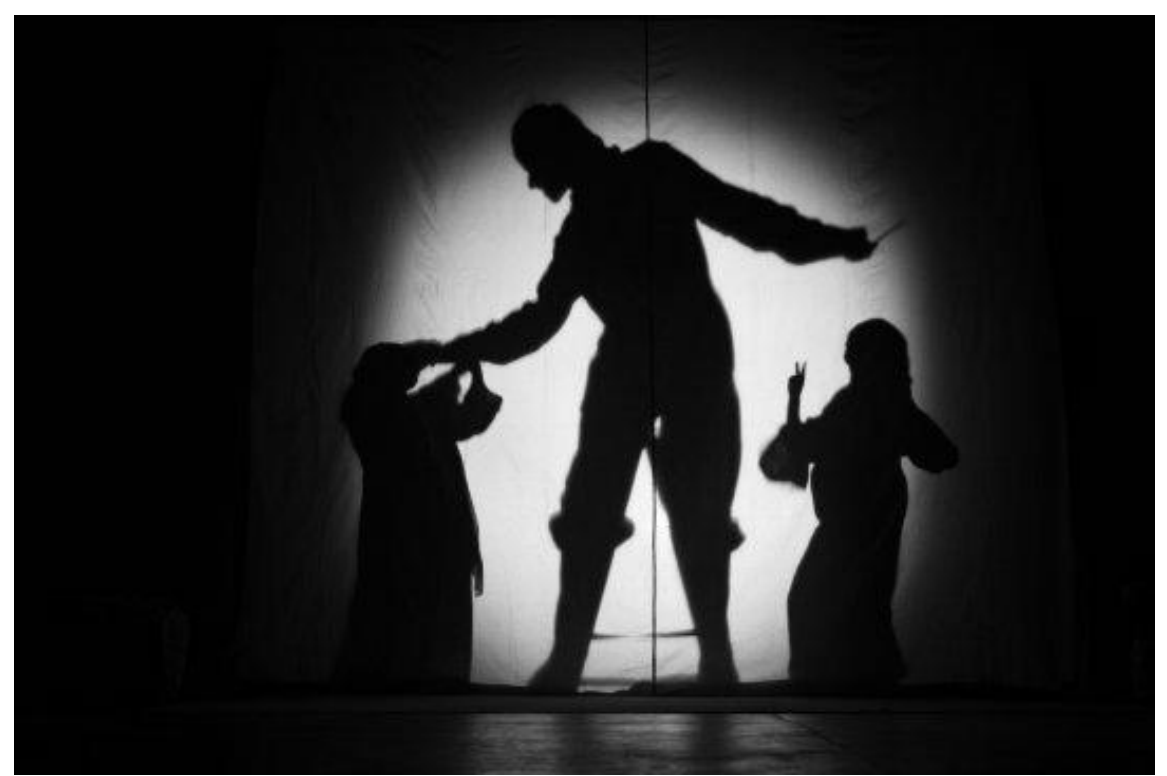

Figure 125: L'Oppression de Jénine, pièce collective montée par le théâtre Ashtar URL : http://www.info-palestine.net/article.php3?id_article=2287

\section{Iraq : un art encore juvénile}

Sulaymân Ghazâla (1853-1929) écrivit La Manière dont les héros parlent (1911), la première pièce irakienne en vers, ainsi que 'Ali Khoja et Droit et justice qui, toutes deux, 
commentent l'actualité socio-politique. Haqqî al-Shiblî fonda une troupe en 1927, juste un an après le passage de George Abyad, se rendit en Égypte pour étudier les arts de la scène auprès de la troupe de Fâtima Rushdî, puis à Paris. Alwân Abû Sharâra écrivit Seule en 1930 avec une introduction qui pose les fondements du théâtre en Iraq. Yahyâ Qâf al-Shaykh 'Abd alWâhid (1900-1996) se servit du théâtre comme arme sociale et intellectuelle à travers des pièces historiques (La Conquête de l'Égypte, La Bataille de Qâdesiyya). Khâlid al-Shawwâf (né en 1924) publia plusieurs pièces versifiées (Shamsu, Les Remparts, L’Olivier).

Le dramaturge iraqien le plus en vue est incontestablement Yûsuf al-'Anî (né en 1927), un auteur préoccupé par les problèmes politiques et sociaux de façon réaliste ou allégorique. Ses premières pièces, à la langue simple et dépassant rarement un seul acte, traitent la bureaucratie et la corruption (Le Chef de gang, 1951), l'injustice d'un système médical avide d'argent (L’Argent des médicaments, 1952), les pratiques abusives des cliniques privées (Six dirhams, 1954), la persécution des dissidents politiques (Je suis ta mère, Shakir, 1955), les mauvais traitements réservés aux handicapés mentaux (Jamil, 1962). Bienvenue à la vie (1960) décrit la tyrannie du régime qui précéda la Révolution de 1958, et magnifie la cause nationaliste. Une Nouvelle Image (1964) raconte les aventures d'un marchand enrichi prêt à abandonner sa famille pour épouser une très jeune femme. La Clé (1968) met en scène la quête de sens d'un jeune couple, ses échecs et désillusions, le tout entrecoupé de commentaires politiques appelant à l'action et de chants folkloriques. Terrain vague (1970) est un mélange de réalisme et de fantaisie au sein duquel s'affrontent les méchants colonialistes et le peuple innocent, et qui intègre également une critique de la politique américaine au Vietnam et au Proche-Orient et des poèmes d'écrivains palestiniens.

Sa ‘dun al-’Ubaydî (né en 1935) écrivit et mit en scène ses propres pièces, Le Début et Deux corps sous un parapluie. 'Âdil Kadhîm (né en 1939) recourt souvent à des thèmes folkloriques (Juillet fait tinter la clochette, Le Siège, La mort et la cause). Les pièces réalistes et politiques de Muhyî al-dîn Zankana (né en 1940) sont écrites pour la plupart en arabe classique (Le Secret, La Question, Le Pigeon, Cela arrive) (WECT, 1999, IV, 108-111 ; Badawi in Jayyusi-Allen, $1995: 18-19)$.

\section{Le théâtre au Maghreb}

\section{A. Tunisie : le poids écrasant du pouvoir politique}

L'histoire du théâtre tunisien est indissociable de celle des origines du modernisme tunisien : convaincus des potentialités du théâtre en matière d'éducation civique, les Jeunes 
Tunisiens ont joué un rôle primordial dans son acclimatation. Le théâtre tunisien est né sous l'influence des compagnies égyptiennes et levantines en tournée dans le pays. La première troupe tunisienne, baptisée «L’Étoile» (al-Nejma) vit le jour en 1908. Le premier texte dramatique tunisien en arabe littéraire, Le Sultan entre les murs du palais Yildiz de M'hamed Jaïbi $^{4}$ fut monté en 1909. En 1911, l'élite politique forma les troupes de la Dignité (alChahâma) et des Lettres (al-Adâb). L'art dramatique se propagea rapidement dans la capitale et les grandes villes, puis en province.

Dans les années 1920 et 1930, le théâtre tunisien dénonçait surtout les tares de la société traditionnelle : les superstitions, l'obscurantisme, l'ignorance, le fatalisme, le rejet de la modernité. Jusqu'à l'Indépendance, il resta un théâtre amateur aux moyens limités et peinant à se renouveler. On peut discerner deux grandes tendances : le théâtre «historique » glorifie le passé arabe en cherchant à se différencier de l'Occident; le théâtre « social », qui englobe à la fois des traductions d'œuvres européennes et des pièces originales, dénonce les injustices et l'occidentalisation de la société. Ces œuvres ne sont souvent que le reflet des valeurs traditionnelles, ce qui se traduit par une absence de vision critique de l'histoire et un certain conservatisme (Ben Cheikh, Madani, in Corvin, 2008: 1377). Néanmoins, les compagnies tunisiennes se produisirent régulièrement jusqu'en 1956, date de l'Indépendance, et participèrent efficacement à la prise de conscience des masses.

Après l'Indépendance, les choses évoluent avec l'intervention de l'État : en 1953, la municipalité de Tunis fonde une troupe professionnelle ; en 1962, le régime bourguibien crée un ministère des Affaires culturelles. Des étudiants sont envoyés à l'étranger, des troupes sont formées en province, des théâtres sont construits, la création est encouragée, un Festival du Thêâtre arabe est créé en 1964 et catalyse les efforts de définition de cet art dans les pays arabes. Malheureusement, l'État en profite pour contrôler la production grâce à la Commission nationale d'orientation théâtrale dont le rôle officiel est de veiller à la qualité des œuvres et la fonction réelle de censurer toute critique de l'ordre établi.

Cette censure donne lieu, avec le Manifeste des Onze, à une contestation du théâtre officiel incarné par la troupe de Ben Ayed. La forme et le fond du théâtre dominant sont remis en question, et un nouveau mouvement, le «théâtre du patrimoine » voit le jour, avec, pour chef de file, 'Izz al-din al-Madani (1938-) qui publie plusieurs pièces traitant de la révolution populaire : La Révolte du propriétaire de l'âne (1971), Le Voyage de Hallâj (1973), Les

\footnotetext{
4 Pour les noms propres des artistes maghrébins et les titres des cuvres en dialectal, nous avons omis la translittération savante des mots arabes en français afin de conserver l'orthographe usuelle sous laquelle ils ont acquis une notoriété.
} 
Poèmes Zanj (1974) et Notre seigneur, Sultan Hasan al-Hafsi (1977), dans lesquelles il s'inspire de personnages historiques ou issus de la littérature arabe classique. Cette mouvance milite pour une réhabilitation de la culture arabo-musulmane sans toutefois renier les apports de la modernité. Elle laisse la place, dans les années 1970, au Théâtre populaire qui prône un théâtre issu du peuple et pour le peuple, et dont les représentants sont Mohamed Raja Farhat, F. Jaïbi, S. Ayadi, Raouf Ben Amor, Jalila Baccar. Juha et l'Orient en désarroi, la Geste de Mohamed 'Ali Hammi, Borni et Atra, Zazia la hilalienne feront date dans l'histoire du théâtre tunisien.

En 1975, Jaïbi, Jaziri, Baccar, Driss et Masrouki créent la première troupe privée, le Nouveau Théâtre, qui s'en prend violemment aux failles et injustices de la société et du pouvoir (Pluie d'automne, 1980 ; Lem, 1982 ; A'rab, 1986), tout en explorant des techniques inspirées du Théâtre du Soleil. Jebali fonde l'espace El Teatro, lieu du théâtre expérimental, et travaille sur des pièces en langue dialectale (Paroles en jeu, 1980) ou sans paroles (Malédiction). Raja Ben Hammar expérimente un langage gestuel intégrant la danse. Jaïbi concilie qualité et audience avec Comedia, Familia, Les Amoureux du café désert.

Le théâtre tunisien est encore fragile : il reste populiste et comique, repose sur l'effort personnel d'un nombre restreint d'artistes, et les pièces plus ambitieuses ne recueillent l'assentiment que d'une élite intellectelle (Ben Cheikh et Madani in Corvin, 2008 : 13761378).

\section{B. Algérie : multilinguisme, métissage et lutte}

En Algérie, les premières troupes égyptiennes ('Abd al-Qâdir al-Misrî et Sulaymân Qardahî) se produisirent en 1907-1908, bientôt suivies en 1921 par la troupe syro-libanaise de Georges Abyad : elles ne rencontrèrent cependant le succès qu'auprès de l'élite lettrée, car les pièces présentées (La Conquête de l'Andalousie, La Revanche arabe, Salâh al-din Ayyub) utilisaient l'arabe classique et exigeaient une certaine érudition ; le public populaire préférait les spectacles traditionnels (conteurs, jongleurs ou charmeurs de serpents). L'année suivante, une troupe égyptienne présenta deux drames shakespeariens, Jules César et Roméo et Juliette, et réussit à drainer un public plus nombreux grâce aux chants intercalés entre les actes, technique qui sera reprise par de nombreux metteurs en scène algériens par la suite. Des associations théâtrales virent cependant le jour, et des pièces furent jouées en arabe littéraire, attirant un public lettré. La majorité des Algériens restaient cependant indifférents à l'art 
théâtral à cause du manque de familiarité avec ce type de spectacle et de l'éloignement des thèmes abordés de la vie quotidienne (Bereksi in Samrakandi, 2008 : 22).

À partir de 1921, l'Algérien 'Ali Sharif Tahar rédigea des pièces dans un style innovateur sur des thèmes modernes : La Guérison après la souffrance, Passions trahies, Badi' abordent surtout le problème de l'alcoolisme. En 1922, une pièce anonyme, vraisemblablement d'origine égyptienne, Au service de la patrie, fut interdite pour subversion par les autorités coloniales. La même année, Mohamed Mansali fonda une troupe qui joua des sketches comiques, mais aussi des pièces politiques à alibi historique (La conquête de l'Andalousie). Juhâ de Allalou (de son vrai nom Salâli 'Ali, né en 1902), écrite en arabe algérien et basée sur un personnage du patrimoine populaire est représentée en 1926 : cette année marque l'apparition de la langue dialectale sur scène, ce qui élargit le public des représentations théâtrales et marque le véritable démarrage de la production théâtrale en Algérie. Allalou recourait à la structure du conte sans négliger la forme «classique » européenne.

La période entre 1926 et 1939, essentiellement dominée par quatre auteurs (Allalou, Dahmoune, Ksentini, Bachetarzi), est relativement riche en œuvres comiques et didactiques, présentées jusque dans les coins les plus reculés (Cheniki, 2002 : 23), abordant des thèmes comme le mariage, l'alcoolisme, le divorce, le maraboutisme, les coutumes, les traditions, les femmes ignorantes ou trop vite européanisées, l'hypocrisie des dévots, sujets déjà présents dans les sketches.

Rachid Ksentini (1887-1944), acteur, dramaturge et chanteur, est considéré comme le véritable père du théâtre algérien. Il débuta dans le Mariage de Bou Akline d'Allalou et contribua à mettre en scène une trentaine de productions. Il écrivit sur les problèmes sociaux de son temps en dialecte algérois : dans Chez le pharmacien, une femme malade est soignée par l'intermédiaire de son mari chargé d'expliquer ses symptômes puisque les mœurs ne permettent pas à une femme convenable de se faire examiner par un étranger ; Zeghirebban ou les deux mangeurs de haschisch et les fils du roi (1929), Un Trou dans la terre (1931), Que Dieu nous protège! (1933) satirisent la petite bourgeoisie algérienne (Guardi in Samrakandi, $2008: 26-30$ ).

En 1927, l'acteur-chanteur Mahieddine Bachetarzi (1899-1986) se fit connaitre avec L'Ignorant prétendant à la connaissance, le premier succès d'une longue série. La plupart des pièces des années 1920 et 1930 sont une critique sociale des réalités de l'occupation en arabe dialectal ; elles sont destinées au divertissement autant qu'à l'éveil politique. La popularité des spectacles, qui consistent en sketches aux intentions moralisatrices et satiriques 
accompagnés de danses, provoque une réaction de censure de la part des autorités françaises. Ils ont compris (1932) de Bachetarzi est considérée comme la première pièce politique algérienne dénonçant l'exploitation coloniale. Le théâtre social et politique est ensuite représenté par des pièces comme Pour l'honneur, Béni Oui Oui, Les Traîtres.

La Seconde Guerre mondiale voit la mise sous tutelle du théâtre, tandis que de nouvelles personnalités se manifestent (Rouiched, Mohamed Touri, Mustapha Kateb, Abdelhalim Raïs), et que l'on se met à traduire des auteurs étrangers. Des troupes d'amateurs se forment à Alger et dans les grandes villes de province, et le public bourgeois vient au théâtre pour se divertir. Après la guerre, les liens se resserrent entre le milieu du théâtre et ceux qui font campagne pour l'indépendance. Les autorités coloniales s'efforcent de canaliser le théâtre en favorisant la création d'une troupe officielle, dirigée par Bachetarzi et Mustapha Kateb. Un comité gouvernemental, présidé par Ahmed Tewfiq el-Madani (1899-1983), veille à la qualité et à la variété des textes théâtraux.

Mustapha Kateb contribue à faire émerger un théâtre littéraire, libéré de ses liens avec le chant et la danse. Les pièces historiques en arabe classique glorifient le passé glorieux de l'Algérie, à l'exemple de Hannibal de Madani en 1948, de Yughurta de 'Abd al-Rahman Madawi, qui retrace la lutte de la population locale contre la colonisation romaine, Les Faits des Barâmika et La Reine de Grenade d'Ahmed Rida Houhou (1911-1956) qui reflètent la réalité contemporaine à travers la présentation de faits passés. Quelques pièces religieuses présentent des personnages des débuts de l'Islam comme des modèles pour ceux qui luttent pour l'Indépendance : Bilâl de Mohamed al-'Id Âl Khizlif (1904-1979) et la Naissance du prophète d'Abderrahmane El-Djilali. L'auteur le plus prolifique des années 1940 et 1950 fut Mohamed Touri (1914-1959, Docteur Allel, Hier et aujourd'hui, Tu as mauvaise opinion, L'Argent, Les Trois voleurs, Le chanceux).

Dans les années 1950, les pièces devinrent de plus en plus ouvertement révolutionnaires, comme Le Samson algérien ou Longue vie à la fraternité de Moursali. En 1952, le service de l'Éducation populaire chargea Henri Cordereau de la direction d'une équipe chargée d'animer des stages pour les Algériens désireux de mieux connaître le théâtre : ce groupe entreprit des recherches sur les formes populaires et monta des spectacles adaptés au public algérien et recourant au conteur, au mime et à la marionnette. Ould Abderahmane Kaki est considéré comme le représentant de cette école (Cheniki, 2002 : 31).

Lors de la Guerre d'Indépendance, entre 1954 et 1962, le théâtre devint un art du combat, un «théâtre d'urgence » (A. Cheniki). Mustapha Kateb s'exila en Tunisie où il fonda la Compagnie nationale des arts du front de la libération. La nouvelle troupe du FLN monta 
des pièces très engagées : Vers la lumière (1958), Les Enfants de la Casbah (1959) et les Immortels d'Abdelhalim Raïs (1921-1975) sont des pièces violentes et agressives qui expriment les souffrances et le combat du peuple algérien. La direction du FLN cherchait à compléter la formation politique et idéologique des combattants en faisant représenter ces pièces dans les camps, les hôpitaux et les maquis (Cheniki, 2002 : 35). Au même moment, dans les prisons, d'anciens hommes de théâtre célébraient la lutte de libération nationale. Le Séisme (1958) de Henri Kréa, La Mort des despotes (1959) d'Abdallah Roubaïki, Le Sol d'Abi al-'Id Doudou, Rouge l'aube (1969) d'Asia Djebar, Des Voix dans la casbah (1960) de Bouzaher, Naissance et l'olivier (1962) de Mohamed Boudia, Le Corps encerclé et Les Ancêtres redoublent de férocité (1959) de Kateb Yacine sont très remarqués.

Après l'Indépendance, les hommes de théâtre s'interrogent sur leur pratique et sur la manière d'utiliser l'héritage culturel : Abdelkader Ould Adberrahmane Kaki, Mustafa Kateb, Abdelkader Alloula (1939-), Slimane Benaïssa (1943-), Mohamed Boudia (1932-1973) décortiquent les problèmes du théâtre populaire, de l'adaptation et de la création. Certains privilégient l'adaptation, d'autres prônent un retour aux sources et une redécouverte de soi, d'autres encore envisagent une symbiose de ces deux voies. En 1963, les salles de spectacle sont nationalisées et le Théâtre national algérien (TNA) est créé. Certaines œuvres connaissent un grand succès comme 132 ans (1962), le Peuple des ténèbres, L'Afrique avant l'an I et Les Vieux (1963) de Kaki, Hassan Terro, La Ghoule (1966) et Les Concierges (1970) de Rouiched (Ahmed 'Iyâd), ou Deux pièces cuisine d'Abdelkader Safiri. Les expérimentations en français et en arabe littéral font long feu, tandis que le théâtre en dialectal se développe.

À partir de 1970, avec la décentralisation, des théâtres régionaux se constituent à Annaba, Oran, Constantine, Bel Abbès, Béjaia, Batna, Tizi Ouzou, mais la production se tarit et le public se disperse. Le théâtre amateur se soucie avant tout de l'actualité sociale et politique et fait émerger quelques réels talents comme Slimane Benaïssa dont la pièce Va de l'avant, Boualem (1974) oppose deux personnages incarnant des générations différentes et des opinions opposées. Quelques troupes privées furent fondées: Bordj Ménaïl par Omar Fetmouche, le Théâtre populaire par Hassan el-Hassani, El Qalaa par Ziani Chérif Ayad.

Le théâtre d'Abdelkader Alloula (m. 1994) est tout de suite mal vu du pouvoir en place, parce qu'il met en scène les travers du régime et de la société, et parce qu'il utilise la langue populaire, méprisée par un état soucieux d'arabisation. Dans sa première pièce Les Sangsues (1969), il peint les abus de la bureaucratie dans une société prétendument socialiste, et l'exaspération du peuple. Puis il écrit Le Pain (1970), La Folie de Salim (1972, une 
adaptation du Journal d'un fou de Nicolas Gogol), Les Thermes du Bon Dieu (1975), L'Homme est un loup pour l'homme (1976), Les Dires (1980), Les Généreux (1984), Le Voile (1989), Les Pommes (1992), des pièces adressées à une société qui lui semble avoir perdu ses repères $\mathrm{du}$ fait de la colonisation et de l'endoctrinement salafiste. Il réfléchit sur la transposition du théâtre aristotélicien au monde arabe et s'efforçe de créer un nouveau théâtre, enraciné dans le patrimoine et la culture populaires, faisant appel à la tradition de la Khalqa et du Meddah et mélangeant narration et théâtre.

Kateb Yacine, plus connu comme écrivain de langue française, dirige une troupe théâtrale nommée Action culturelle des travailleurs et financée par le Ministère du travail entre 1971 et 1977. Les représentations avaient lieu dans les usines et les écoles, sur les places de villages et dans les maisons de jeunes autant que dans les théâtres. Le texte en arabe populaire était utilisé comme un instrument et les pièces de Yacine ne paraîtront en français qu'en 1999 sous le titre de Boucherie de l'espérance. Lui aussi se revendique héritier de la Khalqa et du Meddah. (Haddad in Samrakandi, 2008: 33 sq). Mohamed, prends ta valise (1974) soulève le problème de l'émigration et de la recherche de moyens de subsistance, prend à parti la religion, et interroge les rapports entre les sexes.

Rouiched et Kaki représentent deux tendances dramatiques très différentes. Rouiched continue en quelque sorte le travail de Bachetarzi et Ksentini : son théâtre a pour vocation de faire rire autour d'un personnage comique mais débrouillard, dont les tribulations illustrent les aléas de la vie quotidienne (Cheniki, 2002 : 44). Kaki réinjecte dans le théâtre certains éléments de la culture populaire tout en faisant de nombreuses expériences s'inspirant d'Artaud, du théâtre de l'Absurde et de Brecht.

Ould Abderrahman Kaki (1934-1995) crée très tôt sa propre troupe, Mesrah elGaragouz, qui fera parler d'elle à partir de l'Indépendance avec des pièces comme Avant théâtre, Le Filet, Le Voyage, L'Antiquaire clair de lune, Le Porteur d'eau et les saints (1966), À chacun son jugement (1967). Aucune des quelque cent pièces qu'il a produites n'a été publiée. Son théâtre est la recherche d'une adaptation de la chanson rurale, avec ses thèmes et ses formes d'expression, à la scène moderne; ses pièces ressemblent à des fresques historiques ou légendaires à la gloire du peuple algérien ; son univers se révèle parfois dur, souvent magique, mais toujours près du quotidien (Guezali in Samrakandi, $2008: 41 s q$ ).

Dans les années 1980, le ton se fait plus caustique avec Le Vendredi, les gazelles sont sorties (1977) et Le Bateau coule (1983) de Benaïssa, Les Arabes ont dit (1983), Le Voleur d'autobus (1987), Les Martyrs reviennent cette semaine (1987) de Taher Ouettar, Le Cri (1989) de Ziani Chérif Ayad (1946-), L’Arc-en-ciel de Malek Bouguermouh (1946-1989), 
Juha a vendu son âne de Nabil Badrân, L'Escargot têtu de Rashid Boudjedra. Réaliste et didactique, ce théâtre se penche sur les difficultés des humbles et cultive la dérision. Le public augmente et la qualité des pièces également, avec l'abandon du réalisme socialiste. Entre 1985 et 1995, les deux grandes tendances sont la création collective et l'adaptation de pièces à la réalité algérienne (Bouzar et Cheniki in Corvin, 2008 : 45-57). Brecht et Piscator sont très prisés. Yacine, Alloula, Kaki, Benaïssa assimilent l'articulation des formes populaires et l'univers dramatique occidental.

L’instabilité politique, les événements de la décennie 1990, la médiocrité des responsables ne facilitent pas la vie du théâtre. La plupart des animateurs de la scène algérienne disparaissent : Kateb Yacine et Mustapha Kateb meurent en 1989, Kaki en 1994, Alloula et Medjoubi sont assassinés par des terroristes, de nombreux auteurs, metteurs en scène et comédiens s'expatrient en France (Agoumi, Benaïssa, Fellag, Ziani Chérif Ayad). À l'orée de l'an 2000, un nouveau genre de représentation apparaît, à la gloire de l'héroïsme guerrier et des personnages historiques, qui ne recueille pas l'assentiment du public (Cheniki, $2002: 47)$.

Les difficultés auxquelles se heurte le théâtre algérien sont nombreuses et largement partagées par les autres pays du Maghreb: absence de stratégie nationale, pesanteurs idéologiques et bureaucratiques, insuffisance du financement, carence de la formation, manque d'auteurs, indifférence du public (Bouzar, Cheniki, in Corvin, 2008 : 57).

\section{Maroc : le folklore contre la censure}

Le théâtre marocain à ses débuts relève davantage de l'activisme politique que de l'activité artistique ; ses débuts coïncident d'ailleurs avec la guerre du Rif (1921-1926). Il est pénétré par un discours nationaliste contre la présence coloniale française et espagnole ; il dénonce l'analphabétisme, l'hypocrisie et l'obscurantisme, la connivence avec l'occupant, le statut inférieur de la femme, le conflit du modernisme avec la tradition. Il exalte le courage et le savoir, tout en s'appuyant sur des figures héroïques ou mythiques de l'histoire arabomusulmane (Harûn al-Rashîd, Tarîq ibn Ziyâd, Salâh al-dîn al-Ayyûbî), et s'exprime en arabe littéraire. Parmi les pièces emblématiques, il faut citer la Victoire de l'innocence de Mohamed al-Zghari, al-Mansûr al-Dhahabî de Mohamed Ibn Chikh, 'Abbâsa de Mehdi Mniai, al-Walîd b. 'Abd al-Malik de Mohamed al-Haddad et l'Orphelin $d u$ désert d'Abdelouahid al-Chaoui (Berrada, 1988 : 66). 
La répression ne se fait pas attendre : à partir de 1944, les autorités coloniales interdisent le théâtre scénique qui se cantonne désormais dans les maisons privées, même s'il existe un théâtre «autorisé » qui imite le théâtre égyptien et monte des classiques européens. Le théâtre radiophonique naît, avec la diffusion en 1947 de Rashid et les Barmakides de Haddad. La troupe de la Radio, constituée en 1949 et animée par Abdellah Chekroune, s'entoure des meilleurs comédiens et comédiennes pour diffuser des pièces en arabe classique, et, à partir de 1950, des sketches en dialectal marocain. Elle s'oriente prudemment vers des sujets non susceptibles de froisser les autorités : scènes de la vie quotidienne, questions de société, valeurs morales et religieuses, épopées historiques, contes merveilleux. Elle adapte aussi des pièces étrangères. (Ouzri, 1997 : 160 sqq).

Pour mieux encadrer cette pratique émergente, le service de la Jeunesse et des Sports crée en collaboration avec les scènes françaises et marocaines des «centres d'expression dramartique » à Casablanca, Rabat, Fès, El-Jadida, Marrakesh. André Voisin accomplit une mission d'animation et de formation pendant six ans, formant entre autres al-Tayyeb alSaddiki, Abdessamad Kenfaoui, Ahmad al-Tayyeb El-Alj, et contribuant à la formation d'une troupe-école qui deviendra la Troupe du Théâtre marocain. Parmi les pièces alors créées, mentionnons Lam'allam 'Azzûz, adaptée par 'Atâ' Wakil et traitant de la condition féminine dans les sociétés traditionnelles, Les Fourberies de Juhâ de Wakil, comédie centrée sur le conflit des générations, Les Balayeurs d'El-Alj, et Bonhomme Misère du même, farce dramatique sur la pauvreté et l'exploitation. Voisin s'intéresse aux traditions autochtones, mais évite les sujets qui fâchent.

En 1955, un bureau des activités culturelles et de l'éducation populaire est ouvert dès le début de l'Indépendance, et un centre d'art dramatique est inauguré en 1959, qui formera bien des auteurs, metteurs en scène ou comédiens et constituera un riche répertoire (Badry et Cheniki in Samrakandi, 2008 : 890-892). Jusqu'au début des années 1970, le théâtre devient uniquement un art de divertissement, à l'écart des luttes politiques et sociales de l'époque. Une des caractéristiques de cette période est le retour au patrimoine et à la mémoire populaire. À partir de ce moment, toute l'histoire du théâtre marocain va être marquée par la dualité : d'une part, un théâtre amateur, libre, engagé, de recherche ou expérimental, d'autre part, un théâtre étatique, composé de fonctionnaires. La décadence du théâtre officiel contraste avec l'épanouissement et la richesse du théâtre amateur jusqu'aux années 1980.

L'administration marocaine s'efforce de limiter la liberté du théâtre amateur en imposant des thèmes aux Festival national et en désignant des modèles consensuels. Ainsi Abdelkrim Berrechid (né en 1943) a été très régulièrement primé au festival à cause de la 
nature de ses textes jugés non polémiques. Berrechid se caractérise par une écriture poétique et des sujets tirés de l'histoire et des légendes anciennes (Karakush, Othello, Les Destriers et la poudre, Les Noces de l'Atlas, Les Gens et les pierres). Il revendique le retour au patrimoine national et arabe comme unique source et origine du théâtre. En mars 1979, il publie le premier manifeste du théâtre de la fête ou «théâtre cérémoniel » (al-Ikhtifâliyya), qui tente de retrouver ce qui a caractérisé les scènes médiévales occidentales avant l'imposition de l'unité de lieu et du temps linéaire et s'efforce de concilier l'héritage arabo-musulman et l'héritage théâtral universel, et de s'inscrire dans un dialogue interculturel (Berrechid in Samrakandi, 2008 : 75-80). Philosophique, voire existentialiste, ce théâtre traite les thèmes de la recherche de l'identité, de la responsabilité et de l'engagement, de l'aliénation (La Natte de Lunja, Antara dans les miroirs brisés).

Parmi les structures affiliées à un organisme public, il faut citer la troupe du théâtre marocain (al-Maâmora), rebaptisée après l'Indépendance troupe du Centre marocain de recherches dramatiques, et placée sous la responsabilité d'Ahmed Tayeb El-Alj et de Farid Ben M'barek. Elle s'adressait aux travailleurs sans être un théâtre de revendication, se contentant de décrire leurs conditions de vie et leurs aspirations, incitant au travail et à la découverte du monde, célébrant les fêtes. Elle présenta des spectacles de Tayyeb Saddiki (né en 1938, L'Inspecteur) et d'El-Alj (né en 1928, L'Héritier, Le Lait des Convives, Qâdi alkhalqa, Wali Allâh). D'autres auteurs, comme Abdessamad Kenfaoui, n'eurent pas les mêmes opportunités ; il existait clairement une orientation politique visant à réduire le théâtre à un simple divertissement facile, sans prétention ni projet artistique. Cette troupe fut dissoute en 1975 et ses membres fonctionnaires mis à la disposition du Théâtre National Mohammed V qui fut repris en main par El-Alj. Après le départ à la retraite de celui-ci en 1992, son disciple Mohamed al-Jam reprit le flambeau, s'orientant vers le thêâtre de boulevard (Ouzri, 1997 : $165 s q q)$.

La troupe du Petit Masque s'adressait surtout aux élèves des écoles primaires et des collèges, et jouissait d'une relative liberté dans le choix des textes et la démarche artistique (Les Tortues, Ophélie n'est pas morte de Nabil Lahlou, La Porte fermée, œuvre collective mise en scène par Abdellatif Dachraoui), ce qu'elle paiera de sa dissolution en 1974 (Ouzri, 1997 : 177 sqq). Mohamed Timoud développe un théâtre expérimental (Cordes, ficelles et cheveux, Il était une fois, Les Chaussures brillantes). Le théâtre d'Ahmad al-Iraqi est contestataire et prend position en faveur des défavorisés (Viandes aux enchères, Entre la vie et l'oubli, Quand le rideau se lève, 'Urwa témoigne et persiste). La création de Mohamed 
Shahraman s'inscrit dans le quotidien (Les Grenouilles noires, Les Nains dans le filet, Défaite des Nombres).

La troupe de Saddiki, d'abord basée au Théâtre municipal de Casablanca, devint de fait une troupe indépendante et prit le nom de Théâtre des Gens (Masrah al-nâs) en 1974 ; elle mit en scène de nombreuses adaptations de pièces étrangères ainsi que des pièces inspirées du patrimoine arabe (Les Poèmes de Sidi 'Abd al-Rahmân al-Mahjub, l'histoire d'un poète itinérant, Les Séances de Badi 'al-Zamân Hamadâni, Le Livre des délectations et du plaisir partagé, consacré au philosophe du $\mathrm{X}^{\mathrm{e}}$ siècle, Abû Hayyân al-Tawhîdî, Les Sept Grains de beauté) (Ouzri, 1997 : 181 sqq).

La troupe Badaoui est une autre compagnie indépendante, dirigée depuis 1953 par Abdelkader Badaoui, auteur, adaptateur, metteur en scène, comédien et directeur de la compagnie. Il créa un théâtre de lutte pour l'indépendance, puis pour la défense des opprimés (L'Ouvrier licencié, Les Victimes de l'injustice, La Lutte des ouvriers, Les Chômeurs,...). Il décrivit la société et les mœurs (L'Hypocrite, Sans conscience, L'Intérêt Général, Les Enfants des rues), mais malheureusement sans aller au fond des choses pour ne pas se montrer trop contestaire vis-à-vis du pouvoir. Il adapta aussi des pièces du répertoire international. Le Théâtre d'Aujourd'hui, créé par Abdelouahed Ouzri en 1987, a pour ambition de faire connaître des auteurs arabes et marocains. Abdelhak Zerouali est une personne-institution ayant créé son théâtre individuel, présentant des pièces avec un seul comédien (Ouzri, 1997 : 186 sqq). Zerouali exprime avec violence les conflits sociaux et contradictions individuelles à travers des tableaux et des collages-montages inspirés de Piscator et de Grotowski.

$* * * * *$

Si le théâtre arabe fait preuve de dynamisme et de créativité, il lutte avec une énergie et une constance étonnantes contre de nombreux obstacles, la censure et le manque de moyens étant sans doute les plus redoutables. Il fait désormais partie du paysage culturel de la plupart des pays arabes et s'attaque courageusement aux problèmes politiques et sociaux contemporains. Il faut espérer que les bouleversements du Printemps arabe permettront de lui concéder la place qu'il mérite et libèreront sa parole.

\section{Bibliographie}

Abul-Naga, Atiah. Les sources françaises du théâtre égyptien. Alger : SNED, 1972. 
Al-Hakim, Tawfiq. Qâlibunâ al-masrahi. Le Caire, 1967.

Al-Kaghghat, Mohamed. Structure de l'écriture théâtrale au Maroc des origines aux années 1980. Casablanca : Al-Thakâfa, 1986.

Al-Ra'i, Ali. Al-Masrah fi al-Watan al-'Arabi. Kuwait : 'Âlam al-Ma'rifa, 1980.

Aziza, Mohamed. Formes traditionnelles du spectacle. Tunis: Société tunisienne de diffusion, 1975.

Aziza, Mohamed. Image et Islam. Paris : Albin Michel, 1978.

Aziza, Mohamed. Le théâtre et l'islam. Alger : SNED, 1970.

Aziza, Mohamed. Patrimoine culturel et création contemporaine en Afrique et dans le monde arabe. Dakar : NEA, 1977.

Badawi, Elsaïd. «Arab Theatre and Language ». The World Encyclopedia of Contemporary Theatre (WECT), vol. 4, The Arab World, edited by Don Rubin. London : Routledge, 1999. 19-24.

Badawi, Muhammad. Modern Arabic Drama in Egypt. Cambridge : Cambridge University Press, 1988.

Baffet, Roselyne. Tradition théâtrale et modernité en Algérie. Paris : L'Harmattan, 1985.

Barakat-Saad, Ahmed. Le thème du pouvoir dans le théâtre arabe. Lille : Lille III, ANRT, 1994.

Ben Halima, Hamadi. Les principaux thèmes du théâtre arabe contemporain de 1914 à 1960. Tunis : Université de Tunis, 1969.

Ben Halima, Hamadi. Les principaux thèmes du théâtre arabe contemporain de 1914 à 1960 . Tunis, 1969.

Ben Halima, Hamadi. Un demi-siècle de théâtre arabe en Tunisie (1907-1957). Tunis : Publications de l'Université de Tunis, 1974.

Bencheneb, Rachid. "La fête religieuse et populaire dans l'Islam » et «L'Islam ». Guy Dumur (dir). Histoire des spectacles, Paris : Gallimard, «La Pléiade », 1965. 199209 ; 490-511.

Berrada, Mohamed. Le théâtre au Maroc : tradition, expérimentation et perspectives. Lille : P.U. du Septentrion, 1998.

Bin Zidan, 'Abd al-Rahmân. As'ilat al-masrah al-'arabi. Casablanca : Dâr al-thaqâfa, 1987.

Cheniki, Ahmed. Algérie. Vérités du théâtre. Oran : Dâr al-Gharb, 2006.

Cheniki, Ahmed. Le théâtre en Algérie, Histoire et enjeux. Aix-en-Provence : Edisud, 2002.

Cheniki, Ahmed. Théâtre algérien. Itinéraires, tendances. Thèse de doctorat nouveau régime, Paris IV, 1993. Consulté le 3 octobre 2011 en ligne sur le site : http://www.limag.refer.org/Theses/Cheniki.htm

Cheniki, Ahmed. Théâtre algérien. La fin d'un malentendu. Oran : Dâr al-Gharb, 2005.

Faik, Ala Yahya. Theatrical Elements in Religious Storytelling of Medieval Islamic Culture. Ann Arbor : University of Michigan, 1986.

Jayyusi, Salma Khadra (dir.). Short Arabic Plays : An Anthology. Northampton : Interlink Books, 2003.

Jayyusi, Salma Khadra ; Allen, Roger (dir.). Modern Arabic Drama : An Anthology. Indiana : University of Indiana, 1995. [Series in Arab and Islamic Studies].

Karam, Raif. « Theatre in Lebanon ». TDR 47, 4, 2003 : 128-143. 
Khouri, Chaki. Le Théâtre arabe de l'absurde. Paris : A. G. Nizet, 1978.

Landau, Jacob M. Études sur le théâtre et le cinéma arabes, traduit de l'anglais par F. Le Cleach. Paris : Maisonneuve et Larose, 1965.

Landau, Jacob M. Studies in the Arab Theater and Cinema. Philadelphia: University of Pennsylvania Press, 1958.

Lane, Edward W. An Account of the Manners and Customs of Modern Egyptians. London : The Society for the Diffusion of Useful Knowledge, 1836.

Manzalaoui, Mahmoud. Arabic Writing Today, 2 vols. Cairo : American Research Centre in Egypt, 1968-1977.

Mniai, Hassan. Le Théâtre marocain, de sa création à la mise en pratique de la représentation. Rabat : Dâr al-Aman, 2002.

Mniai, Hassan. Lecture des parcours du théâtre marocain. Meknès, 2003.

Moreh Shmuel. Live Theatre and Dramatic Literature in the Medieval Arab World. New York : New York University Press, 1992.

Mubarak, Khalid. Arabic Drama: A Critical Introduction. Khartoum, Sudan : Khartoum University Press, 1986.

Najm, Y. M. Al-masrahiyya fi'l-adab al-'arabi al-hadith, 1847-1914. Beyrouth : Dâr Beyrut li’l-tibâ'a wa'l-nashr, 1956.

Omotoso, Kole. «Arabic Drama in North Africa ». Theatre in Africa, edited by Oyin Ogunba and Abiola Irele. Ibadan : Ibadan University Press, 1978.

Ouzri, Abdelwahed. Le théâtre au Maroc. Structures et tendances. Casablanca: Toukbal, 1997.

Reynolds, Dwight Fletcher. Heroic Poets, Poetic Heros: The Ethnography of Performance in Arabic Oral Tradition. Ithaca NY: Cornell University Press, 1995.

Roth, Arlette. Le Théâtre algérien de langue dialectale, 1926-1954. Paris : François Maspero, 1967.

Saïd, K. Le Théâtre au Liban (1960-1995). Beyrouth : Festival International de Baalbeck, 1998.

Salame, Ghassan. Le théâtre politique au Liban (1968-1973). Beyrouth : Dâr al-Mashreq, 1974.

Samrakandi, Habib (dir.). Le théâtre arabe au miroir de lui-même, $\mathrm{n}^{\circ}$ spécial d'Horizons Maghrébins, 58, 2008.

Sa'rawi, 'Abd al-Mu'ti. Al-Masrah al-'arabi al-mu'âsir. Le Caire, 1986.

Slauth, Georg ; Sami, Zubaida (dir). Mass Culture, Popular Culture and Social Life in the Middle East. Boulder CO : Westview Press, 1987.

Taymur, Mahmud. Talâ'i al-masrah al-'arabi. Le Caire : Al-Maktaba al-Namudagiyya, s.d.

Tomiche, Nada (dir.). Le théâtre arabe. Paris : UNESCO, 1969.

Ukla Irsan, 'Ali. Al-zawaher al-masrahiyya 'ind al-'arab. Damas, 1981.

Wannus, Sa'dallâh. Bayânât li masrah 'arabi jadid. Beyrouth : Dâr al-Fârâbi, 1988.

World Encyclopaedia of Contemporary Theatre, IV The Arab World. London : Routledge, 1999 : articles "Algeria" , 44-56 (Abdallah El-Rubaïki), "Egypt", 70-100 ('Abd al'Aziz Hammouda), "Iraq", 103-119 (A. Fiâd al-Mufraji, S. 'Abd al-Hamid, 'A. Kamal aldin et al.), "Lebanon", 137-159 (Nabil Abu Murad), "Palestine”, 186-197 (Faysal 
Darraj), "Syria", 234-248 (S. Wannus et Nadim Mohammed), "Tunisia", 251-261 (Izzidine al-madani). 